\title{
A bibliography and categorization of bony fishes exhibiting parental care
}

\author{
LAWRENCE S. BLUMER
}

Division of Biological Sciences and Museum of Zoology, University of Michigan, Ann Arbor, Michigan 48109, U.S.A.

\begin{abstract}
Parental care occurs in a diversity of fishes, but predominantly among freshwater groups. Among the approximately 422 families of bony fishes (Osteichthyes), 89 are presently known to exhibit parental care. Grouping these families into eight categories, based on the sex of the care-giver(s), reveals male parental care is as common or more common than female parental care. Although unusual among vertebrates, parental care by males alone is very common among bony fishes. Lists of families, the forms of parental care exhibited, the modes of fertilization, the environments in which reproduction occurs, and the sources of documentation are presented. An extensive bibliography and index are provided.
\end{abstract}

KEY WORDS :-Bony fixhes - Osteichthyes - parental care parental roles - reproductive behaviour.

\section{CONTENTS}

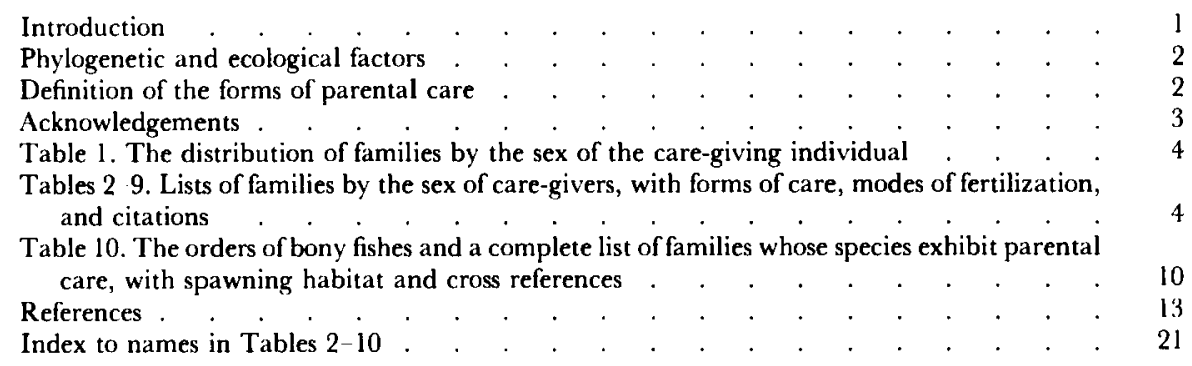

\section{INTRODUCTION}

Parental care, or non-gametic contributions that enhance the survival and reproductive success of the care-giver's offspring, is a diverse phenomenon among fishes. This care manifests itself in a wide variety of activities involving one or both sexes. The vast amount of information on these phenomena has necessitated and encouraged compilations and reviews. One of the earliest such reviews is that of Gill (1906). Numerous researchers have reviewed specific groups of fishes (e.g. Fryer \& Iles, 1972 on cichlids; Wootton, 1976 on sticklebacks); particular 
environments (e.g. Gibson, 1969 on littoral fishes; Mead, Bertelsen \& Cohen, 1964 on deep sea fishes; Reese, 1964 on marine fishes; Sterba, 1963 on freshwater fishes); or specific forms of parental care (e.g. Amoroso, 1960 on internal gestation; Noakes, 1979 on ectodermal feeding; Oppenheimer, 1970 on oral brooding). Balon (1975a) proposed and detailed a scheme of reproductive guilds for fishes based on aspects of reproductive behaviour and ecology. At present, the most authoritative compilation of parental care in fishes is that of Breder \& Rosen (1966). However, Breder \& Rosen's comprehensive work is in need of updating. The information presented here partially fulfills that need.

This paper is based on a literature review concentrating on field studies of fish ecology and behaviour. The occurence of parental care in 89 families of bony fishes $\left(21^{\circ}{ }^{\prime}\right.$ ) of all families) is described by using the systematic classifications of Greenwood, Rosen, Weitzman \& Myers (1966) for teleosts, and Breder \& Rosen (1966) for non-teleosts. These 89 families are categorized into eight mutually exclusive groups based on the sex of the care-giver(s) in Table 1.

\section{PHYLOGENETIC AND ECOLOGICAL FACTORS}

The families characterized by parental care represent a wide variety of groups. These families belong to 21 of the 36 orders of bony fishes. Furthermore, complex care-giving behavior can be found among the most primitive families (Latimeriidae, Lepidosirenidae, Amiidae) as well as the most advanced (Blenniidae, Gobiidae, Belontiidae). Notably, the members of the 'superorders' Elopomorpha and Clupeomorpha do not exhibit any parental care. Ecologically, parental care is predominantly a freshwater phenomenon. Among the 89 families characterized by parental care, reproduction in the care-giving species of 50 families occurs exclusively in fresh water. In 32 families, reproduction is in marine, marine intertidal, or estuarine habitats. The remaining seven families have some care-giving species that reproduce in fresh water and other care-giving species that reproduce in marine, marine intertidal, or estuarine habitats. Considering the 15 orders ( 72 families) in which no parental care is known for any species, the species in 64 families reproduce exclusively in marine or marine intertidal habitats. The species in four families reproduce in fresh water. The remaining four families have some species that reproduce in fresh water and other species that reproduce in marine habitats. The habitat information for the families in these 15 orders was taken from Nelson (1976).

\section{DEFINITION OF THE FORMS OF PARENTAL CARE}

The forms of parental care known to occur in the bony fishes are listed and defined below. The order in which these forms of care are listed corresponds to how frequently each form occurs (Blumer, 1979).

Guarding. Displaying toward and/or actively chasing conspecifics and heterospecifics that approach the eggs or fry, or the site where they are located.

Nest building and maintenance. Digging a depression in the substrate, a burrow, or making an elevated mound with substrate materials. Assembling a cup or tube structure with pieces of vegetation. Blowing mucus-covered bubbles that form a floating mass. All these structures are used to hold eggs and/or fry.

Substrate cleaning. The removal of detritus, algae, and animals from the site where eggs are to be deposited. 
Fanning. Moving the pectoral, pelvic, anal, or caudal fins over the egg mass or fry, thereby aerating them and removing sediment. Aeration and removal of sediment may also be accomplished by forcing water over the eggs or fry through the mouth or gill cavities.

Internal gestation. Female retains eggs in her body and their development takes place inside the ovaries or oviducts.

Removal. Dead or diseased eggs removed by mouth from the egg mass.

Oral brooding. Holding eggs and fry in the mouth or gill cavities during their development, typically ending with the release of well developed fry.

Retrieval. Taking eggs or fry that fall or stray from the nest or school into the mouth, and returning them to the nest or school.

Cleaning. Taking eggs into mouth, manipulating inside the buccal cavity, and returning them to the site from which they were taken.

External egg carrying. Eggs attached to the parent externally and carried until hatching.

Egg burying. Depositing eggs beneath the substrate surface or covering eggs with substrate material.

Moving. Taking eggs or fry by mouth from one location to another, often from one nest to another.

Coiling. The parent coils its body around the egg mass while guarding them. This guarding posture reduces the eggs' exposure to air at low tide, when the oviposition site is intertidal.

Ectodermal feeding. A specialized mucus produced on the body surface of the parent is used as food by the young fry.

Brood pouch egg carrying. Eggs held in a special sac-like external structure during their development.

Splashing. Splashing water on eggs deposited out of water or eggs exposed at times of low tide.

Nest building and substrate cleaning are primarily pre-fertilization activities, whereas the other forms of parental care are post-fertilization activities. The term 'egg' refers to either unfertilized ova or the embryonic phase of development or both. 'Fry' refers to either the larval or juvenile developmental phases or both (see Balon, 1975b). Internal fertilization, in and of itself, is not considered to be a form of parental care. For example, species in the families Phallostethidae and Neostethidae have internal fertilization, but embryonic development (cleavage) does not begin until the eggs are released by the female (Roberts, 1971; Villadolid \& Manacop, 1934). Neither males nor females attend these embryos. Similar conditions exist for members of the Horaichthyidae (Breder \& Rosen, 1966), and for some members of the Cottidae (Hubbs, 1966) and Characidae (Nelson, 1964). Tables 2-9 list the families, forms of care, and references corresponding to each of the eight categories given in Table 1 . The modes of fertilization among the caregiving species of each family are also given in these Tables. Table 10 lists the 36 orders of bony fishes and the families that are characterized by parental care. Ecological data and cross references to Tables 2-9 are also given in Table 10 .

Financial support was provided by $\mathrm{Mr}$ and $\mathrm{Mrs} \mathrm{N}$. Blumer and $\mathrm{Mr}$ and Mrs R. A. Blumer. I am grateful for the advice, comments, and criticisms of the 
following individuals: E. K. Balon, R. M. Bailey, W. D. Hamilton, R. R. Miller, R. A. Nussbaum, G. R. Smith, G. C. Williams, and my fellow graduate students at the University of Michigan. However, any errors or omissions in this work are my own. I also thank K. F. Keyes for her editorial assistance.

TABLE 1

The distribution of families by the sex of the care-giving individual. The 89 families exhibiting parental care are categorized into eight mutually exclusive groups by the sex of the care-giving individual(s). 'Male alone' indicates that when parental care occurs it is given only by the male. 'Male alone and biparental' means that among some species in a family only the male cares for offspring, while among other species in the same family both male and female give parental care (usually together). The meaning of the notation for the other categories follows in a similar manner.

Table 1. The distribution of families by the sex of the care-giving individual

\begin{tabular}{lc}
\hline \multicolumn{1}{c}{ Care given by } & Number of families \\
\hline Male alone & 36 \\
Male alone and biparental & 5 \\
Male alone and female alone & 7 \\
Biparental & 10 \\
Male alone, female alone, and biparental & 5 \\
Female alone and biparental & 3 \\
Female alone & 16 \\
Sex not known & 7 \\
\hline
\end{tabular}

\section{KEY TO TABLES 2-9}

The symbols below indicate the occurrence of each form of parental care within each family, but do not imply that all species in a family exhibit parental care or all the forms of care listed. See text for definitions of the forms of parental care.

G Guarding eggs $(E)$ or fry $(F)$

$\mathrm{N} \quad$ Nest building and/or substrate cleaning

F Fanning and aeration

IG Internal gestation

RD Removal of dead or diseased eggs

ORB Oral brooding

$R \quad$ Retrieval of eggs $(E)$ or fry (F)
CE Cleaning eggs

EEC External egg carrying

EB Egg burying

M Moving eggs (E) or fry $(F)$

GEC Coiling around eggs while guarding

EF Ectodermal feeding of fry

BP Brood pouch

S Splashing eggs deposited out of water

*, Indicates that the available documentation on the family is poor or questionable.

$\dagger$, Indicates that the full reference can be found in the bibliography of Breder \& Rosen (1966) and is not given in the References here. In cases where a reference was not directly consulted, the source of the secondary citation is indicated by the parenthetical phrase, "as cited by Author, date".

The mode of fertilization for the care-giving species in each family is indicated by (I), Internal fertilization; (E), External fertilization. 
Table 2. Families in which the male alone gives parental care

\begin{tabular}{|c|c|}
\hline Family & Forms of care \\
\hline Lepidosirenidae (E) & $\mathrm{GE}, \mathrm{GF}, \mathrm{N}, \mathrm{F}, \mathrm{GEC}$ \\
\hline Amiidae $(\mathbf{E})$ & GE, GF, N \\
\hline Pantodontidat* $(\mathbf{I})$ & GE \\
\hline Notopteridae* (E) & $\mathbf{G F}, \mathbf{N}, \mathbf{F}$ \\
\hline Erythrinidae $(\mathbf{E})$ & $\mathrm{GE}, \mathrm{N}$ \\
\hline Lebiasinidae (E) & $\mathrm{GE}, \mathrm{F}, \mathrm{RD}, \mathrm{CE}, \mathrm{S}$ \\
\hline Citharinidae* (E) & GE \\
\hline Gymnotidae* (E) & GE \\
\hline Cyprinidae $(\mathbf{E})$ & $\mathrm{GE}, \mathrm{N}, \mathrm{F}, \mathrm{RD}, \mathrm{EB}$ \\
\hline
\end{tabular}

$\begin{array}{ll}\text { Siluridae* (E) } & \text { GE, GF } \\ \text { Clariidae (E) } & \text { GE, N } \\ \text { Plotosidae (E) } & \text { GE, N, F } \\ \text { Callichthyidae (E) } & \text { GE, N }\end{array}$

Loricariidae (E) F, RD, CE, EEC

Batrachoididae (E) GE, N, F, S

Antennariidae (E) EEC

Cyprinodontidae (E) GE, F, RD, EB

Gasterosteidae (E)

GE, GF, N, F, RD, $\mathrm{RE}, \mathrm{RF}, \mathrm{CE}, \mathrm{ME}$.

Aulorhynchidae (E) Syngnathidae $(\mathrm{E})$

Synbranchidae $(\mathrm{F})$

Hexagrammidae (E)
GE, N

EFC, BP
GE, F

References

Bouillon, 1961 ; Budgett, 1901 a ; Carter \& Beadle, 1930a†; Johnels \& Svensson, 1954†

Doan, 1938†; Kelly, 1924†; Reighard, 1900, 1902, 1903

Lastein \& van Deurs, 1973; Sachtleben, $1920 \dagger$ (as cited by Breder \& Rosen, 1966)

Smith, H. M., 1933†, 1933; Southwell \& Prashad, $1919 \dagger$ (as cited by Breder \& Rosen, 1966)

Azevedo \& Gomes, $1942 \dagger$ (as cited by Breder \& Rosen, 1966); Moreira, 1919† (as cited by Breder \& Rosen, 1966)

Coates, 1933b†; Innes, 1956; Krekorian, 1976; Krekorian \& Dunham, 1972; Nelson \& Krekorian, 1976; Schapitz, 1962; Shapiro, $1954 \dagger$

Ahl, 1936† (as cited by Breder \& Rosen, 1966)

Hopkins, 1974

Greeley, 1929†; Hankinson, 1908, 1932†; Hubbs \& Cooper, 1936†; Kryzhanovsky et al., $1951 \dagger$ (as cited by Breder \& Rosen, 1966); McMillan \& Smith, 1974; Miller, 1962; Raney, 1939c $\dagger$, 1940b $\dagger$; Reighard, 1910; Tsukahara, 1954†; Van Duzer, 1939†; Westman, 1938†; Wynne-Edwards, 1932†

Aristotle (as translated by Agassiz, 1856); Breder \& Rosen, 1966

Mookerjee \& Mazumdar, $1950 \dagger$

Lake, 1959†, 1967, 1971

Carbonnier, 1880; Carter \& Beadle, 1931†; Dammerhuber, $1935 \uparrow$ (as cited by Breder \& Rosen, 1966); Hoedeman, 1960; Schubert, 1950†; Vipan, 1886

Azevedo, $1938 \dagger$ (as cited by Breder \& Rosen, 1966); Friswold, 1937a $\dagger$ (as cited by Breder \& Rosen, 1966); Isbrücker, 1971; Menezes, 1949

Arora, 1948†; Breder, 194la†; Clapp, 1899; Gray \& Winn, 1961†; Greene, 1899; Gudger, 1908; Hubbs, 1920a $†$; Moore, 1970; Ryder, 1886

Pietsch \& Grobecker, 1980

Barlow, 1961 ; Echelle, 1973 ; Fletcher, 1958+; Herrmann, 1915†; Innes, 1956; Itzkowitz, 1974; Mertz \& Barlow, 1966; Nieuwenhuizen, 196lc†; Rachow, 1929† (as cited by Breder \& Rosen, 1966); Raney et al., 1953†

Assem, 1967; Breder, 1936a†; Iersel, 1953†, 1958†; Köhler, 1926† (as cited by Breder \& Rosen, 1966); Leiner, 1930† (as cited by Breder \& Rosen, 1966); McInerney, 1969; McKenzie, 1974; McKenzie \& Keenleyside, 1970; Morris, D., 1952†, 1958; Reisman, 1963; Reisman \& Cade, 1967; Rowland, 1974; Seal, $1932 \uparrow$; Steinke, $1920 \dagger, 1921 \dagger$ (as cited by Breder \& Rosen, 1966); Winn, 1960†; Wootton, 1971, 1972; Wunder, 1928 $\uparrow, 1930 \dagger$ (as cited by Breder \& Rosen, 1966)

Limbaugh, 1962†

Fiedler, 1954; Graham, 1939†; Gudger, 1906; Heincke, 1880 (as cited by Breder \& Rosen, 1966); Kujawa, 1960; McCulloch, 1914; Straughan, 1960†; Strawn, 1958†; Takai \& Mizokami, $1959 \dagger$

Lake, 1971; Taylor, 1913; Wu \& Liu, 1942† (as cited by Breder \& Rosen, 1966)

Clemens \& Wilby, 1946†; DeMartini, 1977; Edwards, 1971; Fujita \& Uchida, 1959b† (as cited by Breder \& Rosen, 1966); Wilby, 1937† 
Table 2-continued

\begin{tabular}{|c|c|c|}
\hline Family & Forms of care & References \\
\hline Cottidae (E) & $\mathrm{GE}, \mathrm{N}, \mathrm{F}, \mathrm{RD}, \mathrm{ME}$ & $\begin{array}{l}\text { Bailey, 1952 } \uparrow \text {; DeMartini, 1978a; Downhower \& Brown, } \\
\text { 1977; Elmhirst, 1915; Ennis, 1970; Hann, 1927†; } \\
\text { Ludwig \& Norden, 1969; Morris, 1954†; Peek, 1869; } \\
\text { Savage, 1963; Simon \& Brown, 1943 †; Smith, 1923†; } \\
\text { Smyly, 1957†; Westin, 1969, 1970 }\end{array}$ \\
\hline Cyclopteridae (E) & GE, ORB & $\begin{array}{l}\text { DeMartini, 1978b; Gilbert, 1895; McIntosh, 1886, 1897; } \\
\quad \text { Parr, } 1926 \dagger\end{array}$ \\
\hline Pseudochromidae (E) & GE, N, F & Lubbock, 1975 \\
\hline Plesiopidae* (E) & GE & Mito, $1955 \dagger$ (as cited by Breder \& Rosen, 1966) \\
\hline Theraponidae* $(\mathbf{E})$ & $\mathrm{GE}, \mathrm{F}$ & Randow, 1934a† (as cited by Breder \& Rosen, 1966) \\
\hline Centrarchidae (E) & $\mathrm{GE}, \mathrm{GF}, \mathrm{N}, \mathrm{F}$ & 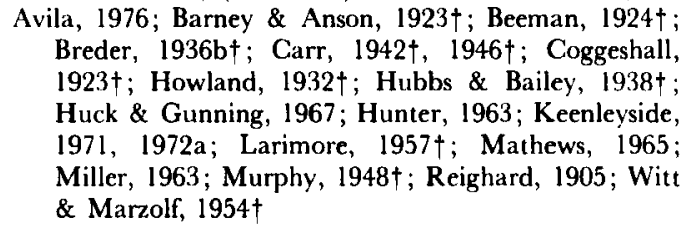 \\
\hline Sparidae (E) & GE, N, F & Hardy, 1959†; Kent, 1883; Penrith, 1972; Wilson, $1958 \dagger$ \\
\hline Nandidae $(\mathbf{E})$ & GE, N, F, RD, RF & $\begin{array}{l}\text { Arnold, 1948a †; Barlow, 1964, 1967; Campbell, 1948b†; } \\
\text { Lauppe, } 1907 \text { (as cited by Breder \& Rosen, 1966) }\end{array}$ \\
\hline Labridae (E) & $\mathrm{GE}, \mathrm{N}, \mathrm{F}$ & $\begin{array}{l}\text { Fiedler, 1964; Potts, } 1974 ; \text { Söljan, 1930a } \dagger, \mathrm{b} \dagger, \mathrm{c} \dagger \text { (as cited } \\
\text { by Breder \& Rosen, 1966); Terry, 1951 } \dagger\end{array}$ \\
\hline Opisthognathidae $(\mathbf{E})$ & GE, N, ORB & Böhlke \& Chaplin, 1957†; Colin, 1971; Leong, 1967 \\
\hline Blenniidae $(\mathrm{E})$ & GE, N, F, RD & 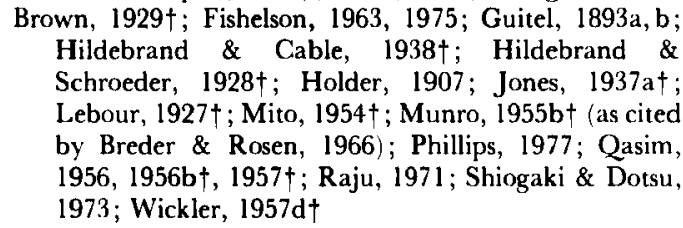 \\
\hline Tripterygiidae (E) & $\mathrm{GE}, \mathrm{F}$ & Graham, 1939†; Ruck, 1973a \\
\hline Kurtidae (E) & EEC & $\begin{array}{l}\text { Guitel, 1913; Hardenberg, 1936†; Lake, 1971; Weber, } \\
\quad 1910\end{array}$ \\
\hline Tetraodontidae (E) & GE, F & $\begin{array}{l}\text { Cohn, } 1912 \text { (as cited by Breder \& Rosen, 1966); } \\
\text { Schreitmüller, 1930at (as cited by Breder \& Rosen, } \\
\text { 1966) }\end{array}$ \\
\hline
\end{tabular}

Table 3. Families with male alone and biparental care

\begin{tabular}{|c|c|c|}
\hline Family & Forms of care & References \\
\hline Gobiesocidae (E) & $\mathrm{GE}, \mathrm{F}$ & $\begin{array}{l}\text { Graham, 1939†; Martin \& Martin, 1970; Ruck, 1971, } \\
\text { 1973b; Runyan, 1962†; Smith, W. A., } 1885\end{array}$ \\
\hline Amphipnoidae* (E) & GE, GF, N, RE & $\begin{array}{l}\text { Das, 1927†; Finckh, 1917†, 1918+ (as cited by Breder \& } \\
\text { Rosen, 1966) }\end{array}$ \\
\hline Pomacentridae (E) & $\begin{array}{l}\mathrm{GE}, \mathrm{GF}, \mathrm{N}, \mathrm{F}, \mathrm{RD}, \\
\mathrm{CE}, \mathrm{EF}\end{array}$ & $\begin{array}{l}\text { Abel, 1961 }+ \text {; Albrecht, 1969; Allen, 1972; Breder \& } \\
\text { Coates, 1933†; Brinley, 1939†; Clarke, 1970; } \\
\text { Fishelson, 1964, 1970a; Fishelson et al., 1974; Fricke, } \\
\text { 1974; Keenleyside, 1972b; Limbaugh, 1964; } \\
\text { Mapstone \& Wood, 1975; Moyer, 1975; Moyer \& } \\
\text { Bell, 1976; Moyer \& Sawyers, 1973; Myrberg et al., } \\
\text { 1967; Robertson, 1973; Russell, 1971; Sale, 1970, } \\
\text { 1971; Swerdloff, 1970; Turner \& Ebert, 1962; } \\
\text { Verwey, 1930† }\end{array}$ \\
\hline Anabantidae (E) & GE, N, RE, RF & $\begin{array}{l}\text { Berns \& Peters, 1969; Forselius, } 1957 \dagger \text {; Nieuwenhuizen, } \\
\text { 1957a†; Rohrbach, 1939c } \dagger \text { (as cited by Breder \& } \\
\text { Rosen, 1966); Peters, 1971 }\end{array}$ \\
\hline
\end{tabular}


Table 3-continued

\begin{tabular}{|c|c|c|}
\hline Family & Forms of care & References \\
\hline Belontiidae (E) & $\begin{array}{l}\text { GE, } N, F, \text { ORB, RE, RF, } \\
\text { CE (only males ORB) }\end{array}$ & 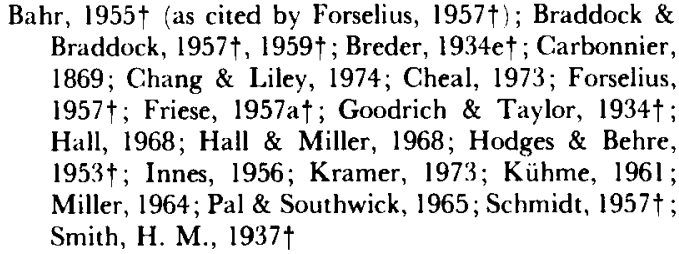 \\
\hline
\end{tabular}

Table 4. Families with male alone and female alone parental care

\begin{tabular}{|c|c|c|}
\hline Family & Forms of care & References \\
\hline Umbridae $(\mathrm{F})$ & $\mathrm{GE}, \mathrm{N}$ & $\begin{array}{c}\text { Breder \& Rosen, 1966; Fowler, 1921†; Hagen et al, 1972; } \\
\text { Landeck, } 1914 \text { (as cited by Breder \& Rosen, 1966) }\end{array}$ \\
\hline Bagridae $(\mathbf{E})$ & GF, N, EEC, EF & $\begin{array}{l}\text { Eggert, } 1930 \dagger \text { (as cited by Breder \& Rosen, 1966); } \\
\text { Sundararaj, } 1962 \dagger\end{array}$ \\
\hline Ariidae $(E)$ & ORB & $\begin{array}{l}\text { Dmitrenko, 1970; Evermann \& Goldsborough, 1902; } \\
\text { Gudger, 1916†, 1918†; Gunter, 1947†; Hubbs \& } \\
\text { Miller, 1960†; Lake, 1971; Lake \& Midgley, 1970a; } \\
\text { Lee, 1931 }+1937 \dagger ; \text { Merriman, 1940†; Pantulu, 1963 }\end{array}$ \\
\hline $\begin{array}{l}\text { Apogonidae }(\mathbf{E}) \\
\text { (I in one species with } \\
\text { male parental care) }\end{array}$ & GE, ORB & $\begin{array}{l}\text { Ebina, 1932a } \dagger \text {; Fishelson, 1970b; Garnaud, 1950b } \dagger, 1962 \text {, } \\
\text { 1963; Petit, 1931 } \dagger \text {; Rudel, 1934† (as cited by Breder } \\
\text { \& Rosen, 1966); Sakamoto, 1930†; Smith, C. L. et al., } \\
\text { 1971 }\end{array}$ \\
\hline Percidae (E) & GE, F, RD, EB & $\begin{array}{l}\text { Adams \& Hankinson, 1928†; Atz, 1940†; Constantz, } \\
\text { 1979; Lake, 1936†; Raney \& Lachner, 1939†; } \\
\text { Reeves, 1907; Winn, 1953†, 1958a }+, \mathrm{b}^{\dagger}\end{array}$ \\
\hline $\begin{array}{l}\text { Clinidae }(\mathbf{E}) \\
\text { (I only in species with } \\
\text { IG })\end{array}$ & $\begin{array}{l}\mathrm{GE}, \mathrm{N}, \mathrm{F}, \mathrm{IG}, \mathrm{RE}, \mathrm{CE} \\
\text { (female care limited to } \\
\mathrm{IG})\end{array}$ & $\begin{array}{l}\text { Al-Uthman, } 1960 \dagger \text {; Breder, 1939c } \dagger, 1941 \mathrm{~b} \dagger \text {; Fusari, } 1895 \\
\text { (as cited by Breder \& Rosen, 1966); Guitel, 1892a, } \\
\text { 1893a; Hubbs, 1952†; Jillett, 1968; Smith, 1950† }\end{array}$ \\
\hline Stichaeidae (E) & $\mathrm{GE}, \mathrm{F}, \mathrm{ME}, \mathrm{GEC}$ & $\begin{array}{l}\text { Clemens \& Wilby, 1946†; LeDrew \& Green, 1975; } \\
\text { Marliave \& DeMartini, 1977; Schultz \& DeLacy, } \\
\text { 1932†; Slipp, 1948a† (as cited by Breder \& Rosen, } \\
\text { 1966); Wourms \& Evans, } 1974\end{array}$ \\
\hline
\end{tabular}

Table 5. Families with biparental care

\begin{tabular}{|c|c|c|}
\hline Family & Forms of care & References \\
\hline Galaxiidae (E) & $\mathbf{N}$ & Cadwallader, 1976 \\
\hline Heteropneustidae (E) & GE & Holly, $1928 \dagger, 1931 \dagger$ (as cited by Breder \& Rosen, 1966) \\
\hline Aphredoderidae* (F) & GE, $N$ & Eddy \& Underhill, 1974 \\
\hline Channidae (E) & $\mathrm{GE}, \mathrm{GF}, \mathrm{N}$ & $\begin{array}{l}\text { Alikunhi, 1953; Chacko \& Kuriyan, 1947†; } \\
\text { Deraniyagala, 1929†; Herre, 1924†; Khan, 1924†; } \\
\text { Qayyum \& Qasim, 1962; Raj, 1916† (as cited by } \\
\text { Breder \& Rosen, 1966); Swift, 1935†; Willey, 1909a; } \\
\text { Yapchiongco, } 1963\end{array}$ \\
\hline Alabetidae* (E) & GE & Haswell, 1890 \\
\hline Serranidae* $(\mathbf{E})$ & GE, N & $\begin{array}{l}\text { Dakin \& Kesteven, } 1938+\text { (as cited by Breder \& Rosen, } \\
\text { 1966); Lake, } 1959 \dagger\end{array}$ \\
\hline Nototheniidae $(\mathrm{E})$ & GE, N, F & Daniels, 1978,1979 \\
\hline Anarhichadidaet $(\mathrm{E})$ & GE, $\mathbf{N}$, GEC: & Penfold, 1966 \\
\hline Osphronemidae (E) & GE, GF, N, F, RE & $\begin{array}{l}\text { Bhimachar et al., 1944†; Gill, 1874; Kulkarni, 1943†; } \\
\text { Roxas \& Umali, 1937† }\end{array}$ \\
\hline Balistidae (F) & GF, N, F & Garnaud, 1960 \\
\hline
\end{tabular}


Table 6. Families with male alone, female alone, and biparental care

\begin{tabular}{cll}
\hline Family & Forms of care & References \\
\hline
\end{tabular}

Osteoglossidae (E) GE, GF, N, ORB Budgett, 1901b; Fontenele, $1959 \uparrow$ (as cited by Breder \&
(care by female alone $\quad$ Rosen, 1966); Lake, 1971; Lake \& Midgley, 1970b; limited to ORB) Luling, 1968; Menezes, 1951 $\dagger$ (as cited by Breder \& Rosen, 1966); Oliveira, 1944† (as cited by Breder \& Rosen, 1966); Scott \& Fuller, 1976; Smith, 1931†, 1945†; Svensson, $1933 \dagger$

Characidae* (E) GE, GF, N, F, RD, ME Breder \& Rosen, 1966; Braker, 1960, 1963; Budgett, 1901b; Innes, 1956; Ledecky, 1966; Svensson, 1933†

Ictaluridae (E) $\quad \mathrm{GE}, \mathrm{GF}, \mathrm{N}, \mathrm{F}, \mathrm{CE}$

Breder, 1935a $\dagger, 1939 b \dagger ;$ Clemens \& Sneed, 1957†; Evcleshymer, 1901; Fontaine, 1944†; Fowler, 1917†; Smith \& Harron, 1904; Taylor, 1969; Wallace, 1972

Cichlidae (E)

GE, GF, N, F, RD, ORB, Aronson, 1948†, 1949†; Atz, 1954†, 1959†; Baerends \& RF, CE, ME, MF, EF Baerends-van Roon, 1950†; Barlow, 1974; Baylis, 1974; Breder, 1934c†; Breder \& Rosen, 1966; Bruton \& Boltt, 1975; Burchard, 1965; Cichocki, 1977; Coe, 1966; Coeckelberghs, 1975; Dünnebier, 1932† (as cited by Breder \& Rosen, 1966); Elder, 1960; ElZarka, 1956†; Evans, 1952†; Fishelson \& Heinrich, 1963; Fryer, 1960at; Fryer \& Iles, 1972; Gordon, 1958†; Graham, 1927†; Greenberg, 1961, 1963; Greenwood, 1956b†; Heinrich, 1967; Hildemann, 1959†; Iles \& Holden, 1969; Keenleyside \& Prince, 1976; Kuenzer, 1957† (as cited by Breder \& Rosen, 1966 ) ; Liebman, 1933†; Lowe, 1956a†, 1959†; LoweMcConnell, 1969; McKaye, 1977a, b; Myrberg, 1965 ; Noakes \& Barlow, 1968, 1973a, b; Noble \& Curtis, 1939; Perrone, 1978; Reid \& Atz, 1958†; Reinboth, $1956 \dagger$ (as cited by Breder \& Rosen, 1966); Ribbink, 1971; Ringelberg, 1935† (as cited by Breder \& Rosen, 1966); Ruwet, 1962, 1963; Sawaya \& Maranhão, 1946†; Skipper \& Skipper, 1956 $\dagger, 1957 \dagger$ (as cited by Breder \& Rosen, 1966); Timms \& Keenleyside, 1975; Ward \& Barlow, 1967; Ward \& Wyman, 1975, 1977; Welcomme, 1967; Werner, 1976; Whitehead, 1962; Wickler, 1962†, 1962, 1965, 1969

Gobiidae (E) GE, N, F, IG, RD (I only in species with IG)
Anderson et al., 1971; Auty, 1978; Breder, 1942†, 1943a $\dagger$; Buen, 1923 $\dagger$ (as cited by Breder \& Rosen, 1966); Dôtu, 1954†, 1955c $\dagger, 1957 \mathrm{~b} \dagger, \mathrm{d} \uparrow, 1958 \mathrm{a} \dagger, \mathrm{d} \dagger, \mathrm{e}$; Ebert \& Turner, 1962; Gale, 1914; Gibson, 1970; Guitel, 1892b, 1895; Hamlyn-Harris, 1931†; Harms, $1935 \dagger$ (as cited by Breder \& Rosen, 1966); Holt, 1899 ; Ishikawa \& Nakamura, $1940^{\dagger}$ (as cited by Breder \& Rosen, 1966); Jones, 1937a†; Lake, 1959†, 1967; Lassig, 1976; Lozano Rey, 1935† (as cited by Breder \& Rosen, 1966); McDowall, 1965; MacGinitie, 1939†; Mashiko, 1976; Mayer, 1929a $\dagger$ (as cited by Breder \& Rosen, 1966) ; Nelson, 1928†; Ninni, 1938† (as cited by Breder \& Rosen, 1966); Riedel, 1914 (as cited by Breder \& Rosen, 1966); Senta \& Wada, 1970; Tavolga, 1950†, 1954†; Weisel, G. F., Jr, 1947† 
Table 7. Families with female alone and biparental care

\begin{tabular}{|c|c|c|}
\hline Family & Forms of care & References \\
\hline Salmonidae (E) & $\begin{array}{l}\text { GE, N, EB } \\
\text { (males aid in EB only) }\end{array}$ & $\begin{array}{l}\text { Babcock, 1931 } \dagger \text {; Belding, 1934a } \dagger \text {; Briggs, 1953†; Brown, } \\
\text { 1938 } \dagger \text {; Fabricius \& Gustafson, 1955 †; Frost, 1965; } \\
\text { Greeley, 1932†; Needham, 1961; Schultz, 1938†; } \\
\text { Schultz \& students, 1935 ; Shapovalov \& Taft, } \\
\text { 1954†; Tautz \& Groot, } 1975\end{array}$ \\
\hline $\begin{array}{l}\text { Zoarcidae (E) } \\
\text { (I only in species with } \\
\text { IG) }\end{array}$ & GE, IG & $\begin{array}{l}\text { Abu Gideiri, 1968; Bretschneider et al., 1947†; Bridge, } \\
\text { 1904; Chute, 1936† (as cited by Breder \& Rosen, } \\
\text { 1966); White, } 1939 \dagger\end{array}$ \\
\hline Pholididae (E) & GE, GEC & $\begin{array}{l}\text { Ehrenbaum, } 1904 \text { (as cited by Gudger, 1927 } \dagger \text { ); Gudger, } \\
\text { 1927†; Holt, 1893; Metz, } 1912 \text { (as cited by Breder \& } \\
\text { Rosen, 1966); Qasim, 1956a†; Smith, 1887† }\end{array}$ \\
\hline
\end{tabular}

Table 8. Families in which the female alone gives parental care

\begin{tabular}{|c|c|c|}
\hline Family & Forms of care & References \\
\hline Latimeriidae (I) & IG & Smith, C. L. et al., 1975 \\
\hline Osmeridae (E) & EB & $\begin{array}{c}\text { Templernan, 1948 } \dagger \text { (as cited by Breder \& Rosen, 1966); } \\
\text { Thompson, 1919; Thompson \& associates, 1936 } \dagger\end{array}$ \\
\hline Aspredinidae (E) & EEC & Breder \& Rosen, 1966; Wyman, 1859 \\
\hline Amblyopsidae (E) & ORB & Eigenmann, 1909 \\
\hline Ophidiidae (I) & IG & $\begin{array}{l}\text { Cohen \& Nielsen, 1972; Nielsen \& Cohen, 1973; Nielsen } \\
\text { et al., 1968; Parr, 1933†; Turner, 1946b†; Wourms \& } \\
\text { Bayne, 1973; Wourms \& Cohen, } 1975\end{array}$ \\
\hline Exocoetidae $(\mathbf{I})$ & IG & Breder \& Rosen, 1966; Scheidnass, 1956†; Weed, $1933 \dagger$ \\
\hline Oryziatidae $(\mathbf{E})$ & EEC & $\begin{array}{l}\text { Amemiya \& Murayama, 1931†; Blanco, 1947b†; Jones, } \\
\text { 1937a } \dagger \text {; Raj, 1916 } \dagger \text { (as cited by Breder \& Rosen, } \\
\text { 1966); Solberg, 1942† }\end{array}$ \\
\hline Adrianichthyidae (I) & IG & Weber \& Beaufort, $1922 \dagger$ \\
\hline Goodeidae (I) & IG & Fitzsimons, 1976; Turner, 1940d $†$ \\
\hline Anablepidae (I) & IG & Eigenmann, 1894; Turner, $1940 \mathrm{~b} \dagger$ \\
\hline Jenynsiidae (I) & IG & Breder \& Rosen, 1966; Turner, 1940e† \\
\hline Poeciliidae (I) & IG & $\begin{array}{l}\text { Baird, 1968, 1973; Clark \& Aronson, 1951†; Constantz, } \\
\text { 1975; Depeche, 1976; Gordon, 1955 }+ \text {; Kunz, 1971; } \\
\text { Rosen \& Bailey, 1963†; Rosen \& Gordon, 1953†; } \\
\text { Turner, 1940a } \dagger\end{array}$ \\
\hline Solenostomidae (I) & BP & Fishelson, 1966; Willey, 1909b \\
\hline Scorpaenidae (I) & IG & $\begin{array}{l}\text { Amoroso, 1960; Fujita, 1957c } \dagger, 1958 \dagger \text {; Goodchild, 1924† } \\
\text { (as cited by Breder \& Rosen, 1966); Graham, 1939†; } \\
\text { Morris, 1956; Phillips, 1958 } \dagger, 1964 \text {; Rass, 1936 ; } \\
\text { Takai \& Fukunaga, 1971 }\end{array}$ \\
\hline Comephoridae (I) & IG & $\begin{array}{l}\text { Berg, 1965; Dybrowski, 1873, } 1901 \text { (as cited by Breder \& } \\
\text { Rosen, 1966); Tcherniaev, } 1971\end{array}$ \\
\hline Embiotocidae (I) & IG & $\begin{array}{l}\text { Bane \& Robinson, 1970; Carlisle et al., 1960†; } \\
\text { Eigenmann, 1894; Hubbs, 1917†, 1921b†; Ishii, } \\
\text { 1957†, 1960†; Lagios, 1965; Rechnitzer \& Limbaugh, } \\
\text { 1952†; Shaw, 1971; Triplett, 1960; Wiebe, } 1968\end{array}$ \\
\hline
\end{tabular}


Table 9. Families in which the sex of the care-givers is not known

\begin{tabular}{|c|c|c|}
\hline Family & Forms of care & References \\
\hline Polypteridae* (E) & GF & Arnoult, 1966; Kerr, 1907 \\
\hline 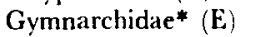 & GE, $\mathbf{N}$ & Budgett, $1901 \mathrm{a}, \mathrm{b}$ \\
\hline Hemiodontidae $(\mathbf{E})$ & GE & Lange, $1934 \dagger$ (as cited by Breder \& Rosen, 1966) \\
\hline Malapteruridae* (E) & ORB & Breder \& Rosen, 1966; Gill, 1906 \\
\hline Carangidae* $\left.^{*} \mathbf{E}\right)$ & GE & Shuleikin, $1958 \dagger$ \\
\hline 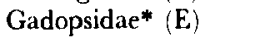 & GE & Jackson, 1978 \\
\hline Luciocephalidae $(\mathbf{E})$ & ORB & Alfred, 1966; Liem, 1967; Tweedie, $1952 \dagger$ \\
\hline
\end{tabular}

TABLE 10

The orders of bony fishes and a complete list of the families with species that exhibit parental care. The manner in which groups of teleost families are listed follows the cladogram of Patterson \& Rosen (1977:120). The number in parentheses after each order is the number of families in that order. The letter symbols that follow each family indicate the habitat in which reproduction occurs for those species that exhibit parental care $(\mathrm{F}=$ fresh water, $\mathbf{M}=$ marine, MIT = marine intertidal or estuarine). These symbols are also used to characterize the families of orders in which no parental care occurs. The 'Care given by' categories correspond to Tables $2-9$ where references for each family are listed.

Table 10. The orders of bony fishes

\begin{tabular}{|c|c|}
\hline Non-teleostean fishes & Care given by \\
\hline \multicolumn{2}{|l|}{ Order Coelacanthiformes (1) } \\
\hline Latimeriidae (M) & Female alone \\
\hline \multicolumn{2}{|l|}{ Order Dipteriformes (2) } \\
\hline Lepidosirenidae $(\mathbf{F})$ & Male alone \\
\hline \multicolumn{2}{|l|}{ Order Polypteriformes (2) } \\
\hline Polypteridae $\langle\mathbf{F}\rangle$ & Sex not known \\
\hline \multicolumn{2}{|l|}{ Order Acipenseriformes (2) } \\
\hline$(2 \mathrm{~F})$ & No parental care \\
\hline \multicolumn{2}{|l|}{ Order Semionotiformes (1) } \\
\hline$(\mathbf{F})$ & No parental care \\
\hline \multicolumn{2}{|l|}{ Order Amiiformes (1) } \\
\hline Amiidae (F) & Male alone \\
\hline Teleostean fishes & Care given by \\
\hline \multicolumn{2}{|l|}{ Osteoglossomorpha } \\
\hline \multicolumn{2}{|l|}{ Order Osteoglossiformes (4) } \\
\hline Osteoglossidae (F) & Female alone and biparental \\
\hline Pantodontidae $(\mathrm{F})$ & Male alone \\
\hline Notopteridae $(\mathrm{F})$ & Male alone \\
\hline \multicolumn{2}{|l|}{ Order Mormyriformes (2) } \\
\hline Gymnarchidae (F) & Sex not known \\
\hline \multicolumn{2}{|l|}{ Elopomorpha } \\
\hline \multicolumn{2}{|l|}{ Order Elopiformes (3) } \\
\hline$(3 \mathbf{M})$ & No parental care \\
\hline \multicolumn{2}{|l|}{ Order Anguilliformes (26) } \\
\hline$(26 \mathrm{M})$ & No parental care \\
\hline \multicolumn{2}{|l|}{ Order Notacanthiformes (3) } \\
\hline$(3 \mathbf{M})$ & No parental care \\
\hline
\end{tabular}


Table 10-continued

\begin{tabular}{|c|c|}
\hline Teleostean fishes & Care given by \\
\hline \multicolumn{2}{|l|}{ Clupeomorpha } \\
\hline \multicolumn{2}{|l|}{ Order Clupeiformes (4) } \\
\hline$(\mathrm{l} F, 2 \mathrm{M}+\mathrm{F}, 1 \mathrm{M})$ & No parental care \\
\hline \multicolumn{2}{|l|}{ Euteleostei } \\
\hline \multicolumn{2}{|l|}{ Order Salmoniformes (37) } \\
\hline Salmonidae $(\mathrm{F})$ & Female alone and biparental \\
\hline Osmeridae (MIT) & Female alone \\
\hline Galaxiidae (F) & Biparental \\
\hline Umbridae $(\mathrm{F})$ & Male alone and female alone \\
\hline \multicolumn{2}{|l|}{ Order Cetomimiformes (9) } \\
\hline$(9 \mathrm{M})$ & No parental care \\
\hline \multicolumn{2}{|l|}{ Order Ctenothrissiformes (1) } \\
\hline$(\mathbf{M})$ & No parental care \\
\hline \multicolumn{2}{|l|}{ Order Gonorynchiformes (4) } \\
\hline$(2 \mathrm{~F}, \mathrm{IF}+\mathrm{M}+\mathrm{MIT}, 1 \mathrm{M})$ & No parental care \\
\hline \multicolumn{2}{|l|}{ Order Cypriniformes (26) } \\
\hline Characidae $(\mathbf{F})$ & Male alone, female alone, and biparental \\
\hline Erythrinidae $(\mathbf{F})$ & Male alone \\
\hline Lebiasinidae $(\mathrm{F})$ & Male alone \\
\hline Hemiodontidae $(\mathbf{F})$ & Sex not known \\
\hline Citharinidae $(\mathbf{F})$ & Male alone \\
\hline Gymnotidae (F) & Male alone \\
\hline Cyprinidae $(\mathbf{F})$ & Male alone \\
\hline \multicolumn{2}{|l|}{ Order Siluriformes $(31)$} \\
\hline Ictaluridae $(\mathbf{F})$ & Male alone, female alone, and biparental \\
\hline Bagridae $(\mathbf{F})$ & Male alone and female alone \\
\hline Siluridae (F) & Male alone \\
\hline Clariidae $(\mathbf{F})$ & Male alone \\
\hline Heteropneustidae (F) & Biparental \\
\hline Malapteruridac $(\mathrm{F})$ & Sex not known \\
\hline Ariidae $(\mathbf{M}+\mathbf{F})$ & Male alone and female alone \\
\hline Aspredinidae (F) & Female alone \\
\hline Plotosidae $(\mathbf{F})$ & Male alone \\
\hline Callichthyidae $(\mathbf{F})$ & Male alone \\
\hline Loricariidae (F) & Male alone \\
\hline \multicolumn{2}{|l|}{ Order Percopsiformes (3) } \\
\hline Amblyopsidae $(F)$ & Female alone \\
\hline Aphredoderidae $(\mathbf{F})$ & Biparental \\
\hline \multicolumn{2}{|l|}{ Order Batrachoidiformes $(\mathrm{l})$} \\
\hline Batrachoididae (MIT) & Male alone \\
\hline \multicolumn{2}{|l|}{ Order Gobiesociformes (1) } \\
\hline Gobiesocidae (MIT) & Male alone and biparental \\
\hline \multicolumn{2}{|l|}{ Order Lophiiformes (15) } \\
\hline Antennariidae $(\mathbf{M})$ & Male alone \\
\hline \multicolumn{2}{|l|}{ Order Gadiformes (10) } \\
\hline Ophidiidae (M) & Female alone \\
\hline Zoarcidae $(\mathbf{M})$ & Female alone and biparental \\
\hline \multicolumn{2}{|l|}{ Order Atheriniformes $(16)$} \\
\hline Exocoetidae $(\mathrm{F})$ & Female alone \\
\hline Oryziatidae $(\mathbf{F})$ & Female alone \\
\hline Adrianichthyidae (F) & Female alone \\
\hline Cyprinodontidae (F) & Male alone \\
\hline Goodeidae $(F)$ & Female alone \\
\hline Anablepidae ( $\boldsymbol{F}$ ) & Female alone \\
\hline Jenynsiidae $(\mathrm{F})$ & Female alone \\
\hline Poeciliidae $(F)$ & Female alone \\
\hline \multicolumn{2}{|l|}{ Order Beryciformes (12) } \\
\hline$(12 \mathrm{M})$ & No parental care \\
\hline Order Zeiformes (6) & \\
\hline$(6 \mathrm{M})$ & No parental care \\
\hline
\end{tabular}


Table 10-continued

\begin{tabular}{|c|c|}
\hline Teleostean fishes & Care given by \\
\hline \multicolumn{2}{|l|}{ Order Lampridiformes (6) } \\
\hline$(6 \mathrm{M})$ & No parental care \\
\hline \multicolumn{2}{|l|}{ Order Gasterosteiformes (9) } \\
\hline Gasterosteidae $(\mathrm{F}+\mathrm{MIT})$ & Male alone \\
\hline Aulorhynchidae (M) & Male alone \\
\hline Solenostomidae $(\mathbf{M})$ & Female alone \\
\hline Syngnathidae $(\mathbf{M})$ & Male alone \\
\hline \multicolumn{2}{|l|}{ Order Channiformes (I) } \\
\hline Channidae (F) & Biparental \\
\hline \multicolumn{2}{|l|}{ Order Synbranchiformes (3) } \\
\hline Alabetidae (MIT) & Biparental \\
\hline Synbranchidae $(\mathbf{F})$ & Male alone \\
\hline Amphipnoidae (F) & Male alone and biparental \\
\hline \multicolumn{2}{|l|}{ Order Scorpaeniformes (21) } \\
\hline Scorpaenidae $(\mathbf{M})$ & Female alone \\
\hline Hexagrammidae $(\mathbf{M})$ & Male alone \\
\hline Cottidae $(\mathbf{M}+\mathbf{F})$ & Male alone \\
\hline Comephoridae $(\mathbf{F})$ & Female alone \\
\hline Cyclopteridae $(\mathrm{M}+\mathrm{MIT})$ & Male alone \\
\hline \multicolumn{2}{|l|}{ Order Dactylopteriformes (1) } \\
\hline$(\mathbf{M})$ & No parental care \\
\hline \multicolumn{2}{|l|}{ Order Pegasiformes (1) } \\
\hline$(\mathbf{M})$ & No parental care \\
\hline \multicolumn{2}{|l|}{ Order Perciformes (143) } \\
\hline Serranidae $(\mathbf{M})$ & Biparental \\
\hline Pseudochromidae (M) & Male alone \\
\hline Plesiopidae (M) & Male alone \\
\hline Theraponidae (M) & Male alone \\
\hline Centrarchidae $(\mathbf{F})$ & Male alone \\
\hline Apogonidae $(\mathbf{M}+\mathbf{M I T})$ & Male alone and female alone \\
\hline Percidae $(F)$ & Male alone and female alone \\
\hline Carangidae (M) & Sex not known \\
\hline Sparidae (M) & Male alone \\
\hline Nandidae $(F)$ & Male alone \\
\hline Embiotocidae (M) & Female alone \\
\hline Cichlidae $(\mathrm{F}+\mathrm{MIT})$ & Male alone, female alone, and biparental \\
\hline Pomacentridae (M) & Male alone and biparental \\
\hline Gadopsidae (F) & Sex not known \\
\hline Labridae $(\mathbf{M})$ & Male alone \\
\hline Opisthognathidae (M) & Male alone \\
\hline Nototheniidae (M) & Biparental \\
\hline Blenniidae $(\mathbf{F}+\mathbf{M}+\mathbf{M I T})$ & Male alone \\
\hline Anarhichadidae $(\mathbf{M})$ & Biparental \\
\hline Tripterygiidae (MIT) & Male alone \\
\hline Clinidae $(\mathbf{M}+\mathbf{M I T})$ & Male alone and female alone \\
\hline Stichaeidae $(\mathrm{M}+\mathrm{MIT})$ & Male alone and female alone \\
\hline Pholididae (MIT) & Female alone and biparental \\
\hline Gobiidae $(\mathbf{F}+\mathbf{M}+\mathbf{M I T})$ & Male alone, female alone, and biparental \\
\hline Kurtidae $(\mathbf{F}+\mathbf{M I T})$ & Male alone \\
\hline Anabantidae $(F)$ & Male alone and biparental \\
\hline Belontiidae $(F)$ & Male alone and biparental \\
\hline Osphronemidae (F) & Biparental \\
\hline Luciocephalidae $(\mathrm{F})$ & Sex not known \\
\hline \multicolumn{2}{|l|}{ Order Pleuronectiformes $(7)$} \\
\hline$(5 M, 2 M+F)$ & No parental care \\
\hline \multicolumn{2}{|l|}{ Order Tetraodontiformes $(7)$} \\
\hline Balistidae (M) & Biparental \\
\hline Tetraodontidale (M) & Male alone \\
\hline
\end{tabular}




\section{REFERENCES}

ABU GIDEIRI, Y. B., 1968. Obervations on the behaviour of the developing Zoarces viniparus (Zoarcidae). Hydrobiologia, 3I: 6064.

AGASSIZ, L., 1856. The 432nd meeting, November 12, 1856. Proceedings of the American Academy of Arts and Sciences, 3: $325-334$.

ALBRECHT, H., 1969. Behaviour of four species of Atlantic damselfishes from Columbia, South America, (Abudefduf saxatiles, A. taurus, Chromis multilineata, C. cyanea; Pisces, Pomacentridae). Zeilschrift für Tierpsychologie, 26: $662-676$.

ALFRED, E. R., 1966. The fresh-water fishes of Singapore. Zoologische Verhandelingen (Leiden), 78: 1. 68.

ALIKUNHI, K. H., 1953. Notes on the bionomics, breeding and growth of the murrel, Ophicephalus striatus Bloch. Proceedings of the Indian Academy of Science, Section B, 38: 10-20.

ALLEN, G. R., 1972. The Anemonefishes: Their Classification and Biology. Neptune City, New Jersey: Tropical Fish Hobbyist Publications.

AMOROSO, E. C., 1960. Viviparity in fishes. Symposia of the Zoological Society of London, 1: 153-181.

ANDERSON, J. R., LAKE, J. S. \& MACKAY, N. J., 1971. Notes on reproductive behaviour and ontogeny in two species of Hypseleotris (=Carassiops) (Gobiidae: Teleostei). Australian Journal of Marine and Freshwater Research, 22: 139-145.

AR ISTOTLE. History of Animals. Book VI, ch. 13, sections 2-6. (As cited by Agassiz, 1856.)

ARNOULT, J., 1966. Behavior and breeding in the aquarium of Polypterus senegalus. Ichthyologica, the Aquarium Journal, 37: $135-140$.

ASSEM, J. VAN DEN, 1967. Territory in the three-spined stickleback Gasterosteus aculeatus L.: An experimental study in intra-specific competition. Behaviour, Supplement $16: 1-164$.

AUTY, E. H., 1978. Reproductive behaviour and early development of the empire fish Hypseleotris compressus (Eleotridae). Australian Journal of Marine and Freshwater Research, 29: 585-597.

AVILA, V. L., 1976. A ficld study of nesting behavior of male bluegill sunfish (Lepomis macrochirus Rafinesque). American Midland Naturalist, 96: 195-206.

BAIRD, R. C., 1968. Aggressive behavior and social organization in Mollienesia latipinna Le Sueur. Texas Journal of Science, 20: 157176.

BAIRD, R. C., 1973. Aspects of social behavior in Poecilia latipinna (Lesueur). Revista de Biologia Tropical, 21: $399-416$.

BALON, E. K., 1975a. Reproductive guilds of fishes: A proposal and definition. Journal of the Fisheries Research Board of Canada, 32: 821864.

BALON, E. K., 1975b. Terminology of intervals in fish development. Journal of the Fisheries Research Board of Canada, 32: $1663-1670$.

BANE, G. \& ROBINSON, M., 1970. Studies on the shiner perch, Cymatogaster aggregata Gibbons, in upper Newport Bay, California. Wasmann Journal of Biology, 28: 259268.

BARLOW, G. W., 1961. Social behavior of the desert pupfish, Cyprinodon macularius, in the field and in the aquarium. American Midland Naturalist, 65: 339-359.

BARLOW, G. W., 1964. Ethology of the Asian teleost Badis badis. V. Dynamics of fanning and other parental activities, with comments on the behavior of the larvae and postlarvae. Zeitschrift für Tierpsychologie, 21: 99- 123.

BARLOW, G. W., 1967. Social behavior of a South American leaf fish, Polycentrus schomburgkii, with an account of recurring pseudofemale behavior. American Midland Naturalist, 78: 215-234.

BARLOW, G. W., 1974. Contrasts in social behavior between Central American cichlid fishes and coral-reef surgeon fishes. American Zoologist, 14: 9-34.

BAYLIS, J. R., 1974. The behavior and ecology of Herotilapia multispinosa (Teleostei, Cichlidae). Zeitschrift fur Tierpsychologie, 34: $115 \cdot 146$.

BERG, L. S., 1965. Freshwater Fishes of the U.S.S.R. and Adjacent Countries, 3. Jerusalem: Israel Program for Scientific Translations. (Translated from Russian.)

BERNS, S. \& PETERS, H. M., 1969. On the reproductive behaviour of Ctenopoma muriei and Ctenopoma damasi: (Anabantidae). Report of the East African Freshwater Fisheries Research Organization, 1968: 44-50.

BLUMER, L. S., 1979. Male parental care in the bony fishes. Quarterly Review of Biology, 54: $149-161$.

BOUILLON, J., 1961. The lungfish of Africa. Natural History, 70: 62-71.

BRAKER, W. P., 1960. The piranha is a dangerous fish . . . and the Shedd Aquarium has 500 young ones coming on! Aquarium, 29(1): 610 .

BRAKER, W. P., 1963. Black piranhas spawned at Shedd Aquarium. Aquarium, 32(10): $12-14$

BREDER, C. M. \& ROSEN, D. E., 1966. Modes of Reproduction in Fishes. Garden City, New York: Natural History Press.

BRIDGE, T. W., 1904. Fishes. In S. F. Harmer and A. E. Shipley (Eds), The Cambridge Natural History, 7. London: Macmillan.

BRUTON, M. N. \& BOLTT, R. E., 1975. Aspects of the biology of Tilapia mossambica Peters (Pisces: Cichlidae) in a natural freshwater lake (Lake Sibaya, South Africa). Journal of Fish Biology, 7: 423 445.

BUDGETT, J. S., 1901a. On the breeding-habits of some West-African fishes, with an account of the external features in development of Protopterus annectens, and a description of the larva of Polypterus lapradei. Transactions of the Zoological Society of London, 16: 115-136. 
BUDGETT, J. S., $190 \mathrm{lb}$. The habits and development of some West African fishes. Proceedings of the Cambridge Philosophical Society, 11: 102-104.

BURCHARD, J. E., 1965. Family structure in the dwarf cichlid Apistogramma irifasciatum Eigenmann and Kennedy. Zeitschrift für Tierpsychologie, 22: 150162.

CADWALLADER, P. L., 1976. Breeding biology of a non-diadromous galaxiid, Galaxias vulgaris Stokell, in a New Zealand river. Journal of Fish Biology, 8: 157-177.

CARBONNIER, P., 1869. Sur le mode de reproduction d'une espèce de poissons de la Chine. Comples Rendus de l'Académie des Sciences, Paris, 69: 489-491.

CARBONNIER, P., 1880. Sur le Callichthys fasciatus Cuvier. Bulletin de la Société Zoologique de France, 5: 288290.

CHANG, B. D. \& LILEY, N. R., 1974. The effect of experience on the development of parental behavior in the blue gourami, Trichogaster trichopterus. Canadian Journal of Zoology, 52: 1499-1503.

CHEAL, M., 1973. Reproduction in the blue gourami. Journal of Biological Psychology, 15: 511.

CICHOCKI, F, 1977. Tidal cycling and parental behavior of the cichlid fish, Biotodoma cupido. Environmental Biology of Fishes, 1: 159-169.

CLAPP, C. M., 1899. The lateral line system of Batrachus tau. Journal of Morphology, 15: 223-264.

CLARKE, T. A., 1970. Territorial behavior and population dynamics of a pomacentrid fish, the garibaldi, Hypsypops rubicunda. Ecological Monographs, 40: 189-212.

COE, M. J., 1966. The biology of Tilapia grahami Boulenger in Lake Magadi, Kenya. Acta Tropica, 23: 146 . 177.

COECKELBERGHS, V., 1975. Territorial, spawning and parental behaviour of Lamprologus brichardi Poll 1974 (Pisces, Cichlidac). Annales de la Socitté Royale Zoologique de Belgique, 105: 73-86.

COHEN, D. M. \& NIELSEN, J. G., 1972. A review of the viviparous ophidoid fishes of the genus Saccogaster. Proceedings of the Biological Society of Washington, 85: 445-468.

COHN, F., 1912. Tetrodon of. T. cutcutia, seine Pflege und Zucht. Blätter für Aquarien- und Terrarienkunde, 23: 582-585. (As cited by Breder \& Rosen, 1966.)

COLIN, P. L., 1971. The other reef. Sea Frontiers, 17: 160 170.

CONSTANTZ, G. D., 1975. Behavioral ecology of mating in the male gila topminnow, Poeciliopsis occidentalis (Cyprinodontiformes: Poeciliidae). Ecology, 56: 966-973.

CONSTANTZ, G. D., 1979. Social dynamics and parental care in the tesselated darter (Pisces: Percidae). Proceedings of the Academy of Natural Sciences of Philadelphia, 131: 131 138.

DANIELS, R. A., 1978. Nesting behaviour of Harpagifer bispinis in Arthur Harbour, Antarctic Peninsula. Journal of Fish Biology, 12: 465-474.

DANIEL.S, R. A., 1979. Nest guard replacement in the Antarctic fish Harpagifer bispinis: Possible altruistic behavior. Science, $\mathcal{N}, Y$., 205: 831-833.

DEMARTINI, E. E., 1977. "Filialcannibalism" of embryos as a male mating strategy and the adaptive significance of promiscuity in Oxylebius pictus (Pisces: Hexagrammidae). Annual Meeting of the American Society of Ichthyologists and Herpetologists. (Abstract.)

DEMARTINI, E. E., 1978a. Spatial aspects of reproduction in buffalo sculpin, Enophrys bison. Environmental Bialogy of Fishes, 3: $331-336$.

DeMARTINI, E. E., 1978b. Apparent paternal care in Liparis fucensis (Pisces: Cyclopteridae). Copeia, 1978: 537-539.

DEPECHE, J., 1976. Acquisition et limites de l'autonomie trophique embryonnaire au cours du développement du poisson téléostéen vivipare Poecilia reticulata. Bulletin Biologique de la France el de la Belgique, 110: 45-97.

DMITRENKO, Y. M., 1970. Reproduction of the sea catfish [Arius thalassimus (Rupp.)] in the Arabian Sea. Journal of Ichihyology (English Translation of Voprosy Ikhtiologii), 10: 634 641.

DOWNHOWER, J. F. \& BROWN, L., 1977. A sampling technique for benthic fish populations. Copeia, 1977: 403-406.

DYBROWSKI, B. I., 1873. Ueber Comephorus baicalensis Pall. Verhandlungen der Zoologisch-Botanischen Vereins Wien, 23: 475-484; Lotos, 24: 122-124. (As cited by Breder \& Rosen, 1966.)

DYBROWSKI, B. I., 1901. Ueber den Baikalischen "Fettsfisch" Comephorus baicalensis Lac., und den Jungfisch derselben. Kosmos, Lwow, 26: 112-141. (As cited by Breder \& Rosen, 1966.)

EBERT, E. E. \& TURNER, C. H., 1962. The nesting behavior, eggs and larvae of the bluespot goby. California Fish and Game, 48: 249-252.

ECHF.LLE, A. A., 1973. Behavior of the pupfish, Cyprinodon rubrofluviatilis. Copeia, 1973: 6876.

EDDY, S. \& UNDERHILL, J. C., 1974. Northern Fishes: With Special Reference to the Upper Mississippi Valley. Minneapolis: University of Minnesota Press.

EDWARDS, G. D., 1971. Sexual dimorphism in the painted greenling and observation of defense of an egg mass. California Fish and Game, 57: 129-131.

EHRENBAUM, E., 1904. Eier und Larven von Fischen der Deutschen Bucht. III. Fische mit festsitzenden Eiern. Helgoländer Wissenschaftliche Meeresuntersuchungen, 6: 160-165. (As cited by Gudger, 1927.)

EIGENMANN, C. H., 1894. On the viviparous fishes of the Pacific coast of North America. Bulletin of the United States Fish Commission 12 (1892): $381-478$.

EIGENMANN, C. H., 1909. Cave vertebrates of America: A study in degenerative evolution. Publications, Carnegie Institution of Washington, 104: 1-241.

ELDER, H. Y., 1960. The breeding of Tilapia zillii. Report of the East African Freshwater Fisheries Research Organization, 1960: 21-22. 
EL.MHIRST, R., 19I5. Habits of Cottus bubalis. Glasgow Naturalist, 7: 43-46.

ENNIS, G. P., 1970. Reproduction and associated behaviour in the shorthorn sculpin, Myoxocephalus scorpius in Newfoundland waters. Journal of the Fisheries Research Board of Canada, 27: 2037-2045.

EVERMANN, B. W. \& GOLDSBOROUGH, E. L., 1902. A report on fishes collected in Mexico and Central Americal, with notes and descriptions of five new species. Bulletin of the United States Fish Commission, 21(1901): 137-159.

EYCLESHYMER, A. C, 1901. Observations on the breeding habits of Ameiurus nebulosus. American . Naturalist, 35 : $911-918$.

FIEDLER, K., 1954. Vergleichende Verhaltensstudien an Seenadeln, Schlangennadeln und Seepferchen (Syngnathidae). Zeitschrifl für Tierpsychologie, 11:358-416.

FIEDLER, K., 1964. Verhaltensstudien an Lippfischen der Gattung Crenilabrus (Labridae, Perciformes). Źpitschrift für Tierpsychologie, 21: 521 - 591.

FISHELSON, L., 1963. Observations on littoral fishes of Israel. I. Behavior of Blennius pavo Risso (Teleostei, Blenniidae . Israel foumal of Zoology, 12:67 80.

FISHELSON, L., 1964. Observations on the biology and behaviour of Red Sea coral fishes. Bulletin of the Sea Fisheries Research Station, Haifa, 37: 1126.

FISHELSON, L., 1966. Solenostomus cyanopterus Bleeker (Teleostei, Solenostomidae) in Elat (Gulf of Akaba). Israel Journal of Zoology, 15: 95-103.

FISHELSON, L., 1970a. Behaviour and ecology of a population of Abudefduf saxatilis (Pomacentridae, Teleostei) at Eilat (Red Sea). Animal Behaviour, 18: 225-237.

FISHELSON, L., 1970b. Spawning behavior of the cardinal fish, Cheilodipterus lineatus, in Eilat (Gulf of Aquaba, Red Sea). Copeia, 1970: 370371.

FISHELSON, L., 1975. Observations on behaviour of the fish Meiacanthus nigrolineatus Smith-Vanig (Blenniidae) in nature (Red Sea) and in captivity. Australian Journal of Marine and Freshwater Research, 26: 329-341.

FISHELSON, I. \& HEINRICH, W., 1963. Some observations on the mouthbreeding Tilapia galilaea L. (Pisces: Cichlidac). Annals and Magazine of Natural History, (13) 6:507 508.

FISHELSON, L., POPPER, D. \& AVIDOR, A., 1974. Biosociology and ecology of pomacentrid fishes around the Sinai Peninsula (northern Red Sea). Journal of Fish Biology, 6: 119-133.

FITZSIMONS, J. M., 1976. Ethological isolating mechanisms in goodeid fishes of the genus Xenotoca (Cyprinodontiformes, Osteichthyes). Bulletin of the Southern California Academy of Sciences, 75(2):84 99.

FRICKE, H. W., 1974. Oko-Ethologie des Monogamen Anemonenfisches Amphiprion bicinctus (Freiwasseruntersuchung aus dem Roten Meer). Zeitschrift für Tierpsychologie, 36: 429-512.

FROST, W. E., 1965. Breeding habits of Windermere charr Salvelinus willughbii (Günther) and their bearing on speciation of these fish. Proceedings of the Royal Society of London, (B) 163:232-284.

FRYER, G. \& ILES, T. D., 1972. The Cichlid Fishes of the Great Lakes of Africa: Their Biologv and Evolution. Edinburgh: Oliver \& Boyd.

FLSARI, R., 1895. Sulle prime fasi di sviluppo dei teleostei. Atti della Accademia dei Lincei, (7) 4: 149-198. (As cited by Breder \& Rosen, 1966.)

GALE, A., 1914. Notes on the breeding-habits of the purple-striped gudgeon, Kreffitus adspersus, Castelnau. Australian Zoologist, 1: 25 - 26

GARNAUD, J., 1960. La ponte, l'eclosion, la larve du baliste Balestes capriscus Linné 1758. Bulletin de l'Institut Océanographique de Monaco, 1169: 16.

GARNAUD, J., 1962. Monographie di l'Apogon mediterranécn, Apogon imberbis (Linné) 1758. Bulletin de l'Institut Océanographique de Monaco, 1248: 183.

GARNAUD, J., 1963. Ethologie d'un poisson extraordinaire: Apogon imberbis. ler Congres International D'Aquariologie, Monaco, 1960, Communications, /D: 51-60.

GIBSON, R. N., 1969. The biology and behaviour of littoral fish. Annual Review of Oceanography and Marine Biology, 7: $367-410$.

GIBSON, R. N., 1970. Observations on the biology of the giant goby Gobius cobitis Pallas. Journal of Fish Biology, 2: $281 \cdots 288$

GILBERT, C. H., 1895. The ichthyological collections of the steamer Albatruss during the years 1890 and 1891. Report of the United States Fish Commission, 19(1893): 393476.

GILL, T. N., 1874. Natural and economic history of the gourami. Report of the United States Fish Commission, $2(1892 / 1893): 710-728$.

GILL, T. N., 1906. Parental care among fresh-water fishes. Report of the Smithsonian Institution, 1905: 403-531.

GREENBERG, B., 1961. Spawning and parental behavior in female pairs of the jewel fish, Hemichromis bimaculatus Gill. Behaviour, 18: 4461 .

GREENBERG, B., 1963. Parental behaviour and recognition of young in Cichlasoma biocellatum. Animal Behaviour, II: 578582

GREENE, C. W., 1899. The phosphorescent organs in the toadfish, Porichthys notatus Girard. Journal of Morphology, 15: 667696.

GREENWOOD, P. H., ROSEN, D. E., WEITZMAN, S. H. \& MYERS, G. S., 1966. Phyletic studies of teleostean fishes, with a provisional classification of living forms. Bulletin of the American Museum of Natural History, 131: 339455. 
GUDGER, E. W., 1906. The breeding habits and the segmentation of the egg of the pipefish (Siphostoma foridae). Proceedings of the United States National Museum, 29: 447-500.

GUDGER, E. W., 1908. Habits and life history of the toadfish (Opsanus tau). Bulletin of the United Siales Bureau of Fisheries, 28: 1071-1109.

GUITEL, F., 1892a. Sur les moeurs du Clinus argentatus Cuv. et Val. Comples Rendus de l'Academie des Sciences, Paris, 115: $295-298$.

GUITEL, F., 1892b. Observations sur les moeurs du Gobins minutus. Archives de Zoologie Expérimentale et Générale, (2) 10: 499-555.

GUITEL, F., 1893a. Observations sur les moeurs de trois blenniides Clinus argentatus Blennius montagui et Blennius sphynx. Archives de Zoologie Expérimentale et Générale (3) 1: 325-384.

GUITEL, F., 1893b. Sur les moeurs du Blennius sphynx, Cuv. et Val. et du Blennius montagui, Fleming. Comptes Rendus de l'Académie des Sciences, Paris, 117: 289-291.

GUITEL., F., 1895. Observations sur les moeurs du Gobius ruthensparri. Archives de Zoologie Expérimentale et Générale, (3) 3: $263-288$.

GUITEL, F., 1913. L'appareil fixateur de l'oeuf du Kurtus gulliveri. Archives de Zoologie Expérimentale et Générale, 52: $1-11$.

HAGEN, D. W., MOODIE, G. E. E. \& MOODIE, P. F., 1972. Territoriality and courtship in the Olympic mudminnow (Novumbra hubbsi). Canadian Journal of Zoology, 50: 1111-1115.

HALL, D. D., 1968. A quantitative analysis of courtship and reproductive behavior in the paradise fish, Macropodus opercularis (Linnaeus). Zeitschrifl für Tierpsychologie, 25: 834-842.

HALL, D. D. \& MILLER, R. J., 1968. A quantitative study of courtship and reproductive behavior in the pearl gourami, Trichogaster leeri (Bleeker). Behaviour, 32: 70-84.

HANKINSON, T. L., 1908. A Biological Survey of Walnut Lake, Michigan. Lansing, Michigan: Wynkoop Hallenbeck Crawford Company. (Michigan State Board Geological Survey Report for 1907.)

HASWELL, W. A., 1890. On the development of Chilobranchus rufus (Teleastei, Symbranchidae). Report of the Australian Association for the Advancement of Science, 1890: 482-487.

HEINCKE, F., 1880. Die Gobiidae und Syngnathidae der Ostsee nebst biologischen Bemerkungen. Archiv für Nalurgeschichte, 46: $301-354$. (As cited by Breder \& Rosen, 1966.)

HEINRICH, W., 1967. Untersuchungen zum Sexualverhalten in der Gattung Tilapia (Cichlidae, Teleostei) und bei Artbastarden. Zeitschrift für Tierpsychologie, 24: 684-754.

HOEDEMAN, J. J., 1960. Studies on callichthyid fishes. 3. Notes on the development of Callichthys (1) (PiscesSiluriformes). Bulletin of Aquatic Biology, 1: 5372.

HOLDER, C. F., 1907. The nest of the kelp fish. American Naturalist, 41: 587-588.

HOLT, E. W. L., 1893. Survey of fishing grounds, west coast of Ireland, 1890-1891: On the eggs and larval and post-larval stages of teleosteans. Scientific Transactions of the Royal Dublin Society, (2) 5:5-121.

HOLT, E. W. L., 1899. Recherches sur la reproduction des poissons osseux, principalement dans le golfe de Marseille. Anneles du Musée d'Histoire Naturelle de Marseille, Zoologie, 5(2): 1-128.

HOPKINS, C. D., 1974. Electric communication in the reproductive behavior of Sternopygus macrurus (Gymnotoidei). Zeilschrifl für Tierpsychologie, 35: 518-535.

HUBBS, CLARK, 1966. Fertilization, initiation of cleavage, and developmental temperature tolerance of the cottid fish, Clinocottus analis. Copeia, 1966: 29-42.

HUCK, L. L. \& GUNNING, G. E., 1967. Behavior of the longear sunfish, Lepomis megalotis (Rafinesque). Tulane Studies in Zoology, 14: 121-131.

HUNTER, J. R., 1963. The reproductive behavior of the green sunfish, Lepomis cyanellus. Zoologica, New York, 48: 13-24.

ILES, T. D. \& HOLDEN, M. J., 1969. Bi-parental mouth brooding in Tilapia galilaea (Pisces, Cichlidae). Journal of Zoology, London, 158: 327-333.

INNES, W. T., 1956. Exotic Aquarium Fishes. Philadelphia: Innes Publishing Company.

ISBRUCKER, I.J. H., 1971. A redescription of the South American catfish Loricariichthys maculatus (Bloch, 1794), with designation of the lectotype and restriction of its type locality (Pisces, Siluriformes, Loricariidae). Bijdragen tot de Dierkunde, $41: 10-18$.

ITZKOWITZ, M., 1974. The effects of other fish on the reproductive behavior of the male Cyprinodon variegalus (Pisces: Cyprinodontidae). Behaviour, 48: $1-22$.

JACKSON, P. D., 1978. Spawning and early development of the river blackfish, Gadopsis marmoratus Richardson (Gadopsiformes: Gadopsidae), in the McKenzie River, Victoria. Australian Journal of Marine and Freshwater Research, 29: 293-298.

JILLETT, J. B., 1968. The biology of Acanthoclinus quadridactylus (Bloch and Schneider) (Teleostei-Blennioidea). II. Breeding and development. Australian Journal of Marine and Freshwater Research, 19: 9-18.

KEENLEYSIDE, M. H. A., 1971. Aggressive behavior of male longear sunfish (Lepomis megalotis). Zeitschrift für Tierpsychologie, 28: 227-240.

KEENLEYSIDE, M. H. A., 1972a. Intraspecific intrusions into nests of spawning longear sunfish (Pisces: Centrarchidae). Copeia, 1972: 272-278.

KEENLEYSIDE, M. H. A., 1972b. The behaviour of Abudefduf zonatus (Pisces, Pomacentridae) at Heron Island, Great Barrier Reef. Animal Behaviour, 20: 763-774.

KEENLEYSIDE, M. H. A. \& PRINCE, C. E., 1976. Spawning-site selection in relation to parental care of eggs 
in Aequidens paraguayensis (Pisces: Cichlidae). Canadian Journal of Zoology, 54: 2135-2139.

KENT, W. S., 1883. Handbook of the Marine and Freshwater Fishes of the British Islands (including an enumeration of every species). International Fisheries Exhibition Literature Handbooks, Part 1, Number 2. London: Clowes.

KERR, J. G., 1907. The development of Polypterus senegalus Cuv. In J. G. Kerr (Ed.), The Works of John Samuel Budgett: 195-284. Cambridge: Cambridge University Press.

KRAMER, D. L., 1973. Parental behaviour in the blue gourami Trichogaster trichopterus (Pisces, Belontiidae) and its induction during exposure to varying numbers of conspecific eggs. Behaviour, 47: 14-32.

KREKORIAN, C. O'N., 1976. Field observations in Guyana on the reproductive biology of the spraying characid, Copeina arnoldi Regan. American Midland Naturalist, 96: 88-97.

KREKORIAN, G. O'N. \& DUNHAM, D. W, 1972. Preliminary observations on the reproductive and parental behavior of the spraying characid Copeina arnoldi Regan. Zeitschrift für Tierpsychologie, 31: 419-437.

KUHME, W., 1961. Verhallensstudien am maulbrutenden (Betta anabatoides Bleeker) und am nestbauenden Kampffisch (B. splendens Regan). Zeitschrift für Tierpsychologie, 18: 33-55.

KUJAWA, S., 1960. Reproduction ethology of Siphonostoma typhli L. [in Polish, English Summary.] Przeglad Zoologiczny, 4: $297-303$.

KUNZ, Y. W., 1971. Histological study of greatly enlarged pericardial sac in the embryo of the viviparous teleost Lebistes reticulatus. Revue Suisse de Zoologie, 78: 187- 207.

LAGIOS, M. D., 1965. Seasonal changes in the cytology of the adenohypophysis, testes and ovaries of the black surfperch, Embiotoca jacksoni, a viviparous percomorph fish. General and Comparative Endocrinology, 5: 207-221.

LAKE, J. S., 1967. Principal fishes of the Murray-Darling River system. In A. H. Weatherley (Ed.), Australian Inland Waters and their Fauna: 192-213. Canberra: Australian National University Press.

LAKE, J. S., 1971. Freshwater Fishes and Rivers of Australia. Melbourne: Thomas Nelson.

LAKE, J. S. \& MIDGLEY, S. H., 1970a. Reproduction of freshwater Ariidae in Australia. Australian fournal of Science, 32: $441 \cdot 442$.

LAKE, J. S. \& MIDGLEY, S. H., 1970b. Australian Osteoglossidae (Teleostei). Australian Journal of Science, 32: $442-443$.

LANDECK, A., 1914. Geschlechtsunterschiede der Zierfische. Blätter für Aquarien- und Terrarienkunde, 25: $49-67$. (As cited by Breder \& Rosen, 1966.)

LASSIG, B., 1976. Field observations on the reproductive behaviour of Paragobiodon spp. (Osteichthyes: Gobiidae) at Heron Island Great Barrier Reef. Marine Behaviour and Physiology, 3: 283-293.

LASTEIN, U. \& VAN DEURS, B., 1973. The copulatory organ of Pantodon buchholzi Peters (Teleostei). Acta Zoologica, Stockholm, 54: 153160.

LAUPPE, L., 1907. Beiträge zur Biologie von Badis badis. Wochenschrift für Aquarien- und Terrarienkunde, 4: 570-571. (As cited by Breder \& Rosen, 1966.)

LEDECKY, E., 1966. Spawning piranhas. Tropical Fish Hobbyist, I4(5): 5-14.

LEDREW, B. R. \& GREEN, J. M., 1975. Biology of the radiated shanny Clvaria subbifarcata Storer in Newfoundland (Pisces: Stichaeidae). Journal of Fish Biology, 7: 485-495.

LEONG, D., 1967. Breeding and territorial behaviour in Opisthognathus aurifrons (Opisthognathidae). Naturwissenschaften, 54: 97.

LIEM, K. F., 1967. A morphological study of Luciocephalus pulcher, with notes on gular elements in other recent teleosts. Journal of Morphology, 121: 103-134.

LIMBAUGH, C., 1964. Notes on the life history of two Californian pomacentrids: garibaldis, Hypsypops rubicunda (Girard), and blacksmiths, Chromis punctipinnis (Cooper). Pacific Science, 18: 41-50.

LOWE-McCONNELL, R. H., 1969. The cichlid fishes of Guyana, South America, with notes on their ecology and breeding behaviour. Zoological Journal of the Linnean Society, 48: 255-302.

LUBBOCK, R., 1975. Fishes of the family Pseudochromidae (Perciformes) in the northwest Indian Ocean and Red Sea. Journal of Zoology, London, 176: 115-157.

LUDWIG, G. M. \& NORDEN, C. R., 1969. Age, growth and reproduction of the northern mottled sculpin (Cottus bairdi bairdi) in Mt. Vernon, Wisconsin. Milwaukee Public Museum Occasional Papers in .Natural History, 2: 1-67.

LULING, K. H., 1968. Arapaima giant fish of Amazonas. Animals, London, 11: 222-225.

McCULLOCH, A. R., 1914. Notes on some Australian pipe-fishes. I. The remarkable sexual dimorphism of Stigmatophora nigra, Kaup. Australian Zoologist, $1: 29-31$.

McDOWALL, R. M., 1965. Studies on the biology of the red-finned bully, Gobiomorphus huttoni (Ogilby). II. Breeding and life history. Transactions of the Royal Society of New Zealand, Zoology, 5: 177-196.

McINERNEY, J. E., 1969. Reproductive behaviour of the blackspotted stickleback, Gasterosteus wheatlandi. Journal of the Fisheries Research Board of Canada, 26: 2061 - 2075.

McINTOSH, W. G., 1886. Notes from the St. Andrews Marine Laboratory (under the Fisheries Board for Scotland). No. V. On the paternal instincts of Cyclopterus lumpus, L. Annals and Magazine of Natural History, (5) 18: $81-84$.

McINTOSH, W. C., 1897. Notes from the Gatty Marine Laboratory, St. Andrews. No. XVII. Annals and Magazine of Natural History, (6) 19: 241-261.

McKAYE, K. R., 1977a. Defense of a predator's young by a herbivorous fish: an unusual strategy. American Vaturalist, 111: $301-315$.

2 
McKAYE, K. R., 1977b. Competition for breeding sites between the cichlid fishes of Lake Jiloá, Nicaragua. Ecology, 58: 291-302.

McKENZIE, J. A., 1974. The parental behavior of the male brook stickleback Culaea inconstans (Kirtland). Canadian Journal of Zoology, 52: 649652.

McKENZIE, J. A. \& KEENLEYSIDE, M. H. A., 1970. Reproductive behavior of ninespine sticklebacks (Pungitius pungitius (L.)) in South Bay, Manitoulin Island, Ontario. Canadian Journal of Zoology, 48: 55-61.

McMILLAN, V. E. \& SMITH, R. F. J., 1974. Agonistic and reproductive behaviour of the fathead minnow (Pimephales promelas Rafinesque). Zeitschrift für Tierpsychologie, 34: 25-58.

MAPSTONE, G. M. \& WOOD, E. M., 1975. The ethology of Abudefduf luridus and Chromis chromis (Pisces: Pomacentridae) from the Azores. Journal of Zoology, London, 175: 179-200.

MARLIAVE, J. B. \& DeMARTINI, E. E., 1977. Parental behavior of intertidal fishes of the stichaeid genus Xiphister. Canadian Journal of Zoology, 55: 60-63.

MARTIN, R. A. \& MARTIN, C. L., 1970. Reproduction of the clingfish, Gobiesox strumosus. Quarterly Journal of the Florida Academy of Sciences, 33: $275-278$.

MASHIKO, K., 1976. Reproductive behavior of an eleotrid goby Odontobutis obscurus in aquaria. Japanese Journal of Ichthyology, 23: 69-78.

MATHEWS, S. B., 1965. Reproductive behavior of the Sacramento perch, Archoplites interruplus. Copeia, 1965: 224-228.

MEAD, G. W., BERTELSEN, E. \& COHEN, D. M., 1964. Reproduction among deep-sea fishes. Deep-Sea Research, 11: 569-596.

MENEZES, R. S. DE, 1949. Incubação labial de ovos pelo macho le "Loricaria typus" Bleeker, da Lagoa do Peixe, Piaui, Brasil (Actinopterygii, Loricariidae, Loricariinae). Revista Brasileira de Biologia, 9(3): 381 - 387

MERTZ, J. C. \& BARLOW, G. W., 1966. On the reproductive behavior of Jordanella foridae (Pisces: Cyprinodontidae) with special reference to a quantitative analysis of parental fanning. Zeitschrift fur Tierpsychologie, 23: 537-554.

METZ, C. W., 1912. The fishes of Laguna Beach, California. Report of the Laguna Marine Laboratory, Pomona College, 1: 19-66. (As cited by Breder \& Rosen, 1966.)

MILLER, H. C., 1963. The behavior of the pumpkinseed sunfish, Lepomis gibbosus (Linnaeus), with notes on the behavior of other species of Lepomis and the pigmy sunfish, Elassoma evergladei. Behaviour, 22: 88-151.

MILLER, R.J., 1962. Reproductive behavior of the stoneroller minnow, Campostoma anomalum pullum. Copeia, 1962: $407-417$.

MILLER, R. J., 1964. Studies on the social behavior of the blue gourami, Trichogaster trichopterus (Pisces, Belontiidae). Copeia, 1964: 469-496.

MOORE, R. H., 1970. Observations on the nest guarding activities of the male Atlantic midshipman, Porichthys porosissimas. Copeia, 1970: 196-197.

MORRIS, D., 1958. The reproductive behavior of the ten-spined stickleback (Pygosteus pungitius L.). Behaviour, Supplement 6:1-154.

MORRIS, R. W., 1956. Early larvae of four species of rockfish, Sebastodes. California Fish and Game, 42: $149-153$.

MOYER, J. T., 1975. Reproductive behavior of the damselfish Pomacentrus nagasakiensis at Miyake-jima, Japan. Japanese Journal of Ichlhyologv, 22: $151-163$.

MOYER, J. T. \& BELL, L. J., 1976. Reproductive behavior of the anemonefish Amphiprion clarkii at Miyakejima, Japan. Japanese Journal of Ichthyology, 23: 23-32.

MOYER, J. T. \& SAWYERS, C. E., 1973. Territorial behavior of the anemonefish Amphiprion xanthurus with notes on the life history. Japanese Journal of Ichthyology, 20:85-93.

MYRBERG, A. A., 1965. A descriptive analysis of the behaviour of the African cichlid fish, Pelmatochromis guentheri (Sauvage). Animal Behaviour, 13: 312-329.

MYRBERG, A. A., BRAHY, B. D. \& EMERY, A. R., 1967. Field observations on reproduction of the damselfish, Chromis multilineata (Pomacentridae), with additional notes on general behavior. Copeia, 1967: 819827.

NEEDHAM, P. R., 1961. Observations on the natural spawning of eastern brook trout. California Fish and Game, 47: 2740.

NEL.SON, J. S., 1976. Fishes of the World. New York: Wiley-Interscience.

NELSON, K., 1964. Behavior and morphology in the glandulocaudine fishes (Ostariophysi, Characidae). University of California Publications in Zoology, 75: 59152.

NELSON, S. G. \& KREKORIAN, C. O'N., 1976. The dynamics of parental care of Copeina arnoldi (Pisces, Characidae). Behavioral Biology, 17: 507-518.

NIELSEN, J. G., JESPERSEN, A. \& MUNK, O., 1968. Spermatophores in Ophidioidea (Pisces, Percomorphi). Galathea Report, 9: 239254.

NIELSEN, J. G. \& COHEN, D. M., 1973. A review of the viviparous ophidioid fishes of the genera Bythites Reinhardt and Abythites new (Pisces, Ophidioidei). Steenstrupia, 3: 7188.

NOAKES, D. L. G., 1979. Parent-touching behavior by young fishes: incidence, function and causation. Environmental Biology of Fishes, 4: 389400.

NOAKES, D. \& BARLOW, G. W., 1968. Parental behavior of Cichlasoma labialum; behavior of the young towards the parents. American Zoologisi, 8: 691. (Abstract)

NOAKES, D. L. G. \& BARLOW, G. W., 1973a. Cross-fostering and parent-offspring responses in Cichlasoma 
citrinellum (Pisces, Cichlidae). Zeitschrift für Tierpsychologie, 33: 147-152.

NOAKES, D. L. G. \& BARLOW, G. W., 1973b. Ontogeny of parent-contacting in young Cichlasoma citrenellum (Pisces, Cichlidae), Behaviour, 46: $221-255$.

NOBLE, G. K. \& CURTIS, B., 1939. The social behavior of the jewel fish, Hemichromis bimaculatus Gill. Bulletin of the American Museum of Natural History, 76: 1- 46.

OPPENHEIMER, J. R., 1970. Mouthbreeding in fishes. Animal Behaviour, 18: 493503.

PAL, B. C. \& SOUTHWICK, C. H., 1965. Reproductive behaviour of the Indian spike-tailed paradise fish, Macropodus cupanus (Cuv. \& Val.). Journal of the Bombay. Natural History Society, 62: 463-474.

PANTULU, V. R., 1963. Studies on the age and growth, fecundity and spawning of Osteogeneiosus militaris (Linn.). Journal du Conseil, Conseil International pour l'Exploration de la Mer, 28: 295-315.

PATTERSON, C. \& ROSEN, D. F., 1977. Review of ichthyodectiform and other Mesozoic teleost fishes and the theory and practice of classifying fossils. Bulletin of the American Museum of Natural Histony, 158: 81-172.

PEEK, R., 1869. Parental attachment of the miller's thumb (Cottus gobio). Student and Intellectual Observer of Science, literature and Art, 3: 455458.

PENFOL.D, V. F., 1966. The wolf-eel. Sea Frontiers, 12: 28.

PENRITH, M. J., 1972. The behaviour of reef-dwelling sparid fishes. Zoologica Africana, 7: 43-48.

PERRONE, M., 1978. Mate size and breeding success in a monogamous cichlid fish. Environmental Biology of Fishes, 3: 193210.

PETERS, H. M., 1971. Sporozoon cysts, the basis of purported "mouth-brooding" in the African labyrinthine fish, Ctenopoma multispinis (Pisces: Anabantidae). Copeia, 1971: 185.

PHIL.IIPS, J. B., 1964. Life history studies on ten species of rockfish (genus Sebastodes). Fish Bulletin, Califarmia Department of Fish and Game, $126: 1 \cdots 70$.

PHIILIPS, R. R., 1977. Behavioral field studies of the Hawaiian rockskipper, Istiblennius zebra (Teleostei, Blenniidae I. Ethogram. Zeitschrift für Tierpsychologie, 43: 122.

PIETSCH, T. W. \& GROBECKER, D. B., 1980. Parental care as an alternative reproductive mode in an antennariid anglerfish. Copeia, 1980: 551 : 553.

POTTS, G. W., 1974. The colouration and its behavioural significance in the corkwing wrass, Crenilabrus melops. Journal of the Marine Biological Association of the United Kingdom, 54: 925-938.

QASIM, S. Z., 1956. Time and duration of the spawning season in some marine teleosts in relation to their distribution. Journal du Conseil, Conseil International pour l'Exploration de la Mer, 21: 144-155.

QAYYUM, A. \& QASIM, S. Z., 1962. Behavior of the Indian murrel, Ophecephalus punctatus, during brood care Copeia, 1962: $465 \cdot 467$.

RAJU, N. S., 1971. Breeding habits, development and life-history of Blennius steindachneri Day from Waltair coast. Proceedings of the Indian Academy of Sciences, (B) 74: 37.45.

REFSE, E. S., 1964. Ethology and marine zoology. Annual Review of Oceanography and Marine Biology, 2: 455488.

REEVES, C. D., 1907. The breeding habits of the rainbow darter (Etheostoma caeruleum Storer), a study in sexual selection. Biological Bulletin, Woods Hole, 14:35 59.

REIGHARD, J. E., 1900. The breeding habits of the dog-fish, Amia calva. Report of the Michigan Academy of Science, Arts and letters, /(1894 1899): 133 137. (Abstract.)

REIGHARD, J. E., 1902. Some further notes on the breeding habits of Amia. Report of the Michigan Academy of Science, Arts and letters, 3(1901): $80-81$. (Abstract.)

REIGHARD, J. F., 1903. The natural history of Amia calva Linnaeus. In G. H. Parker (Ed.), Mark Anniversary I'olume: 57 110. New York: Henry Holt.

RFIGHARD, J. E., 1905. 'The breeding habits, development and propagation of the black bass (Micropterus dolomieu Lacepede and Micropterus salmoides Lacepede). Bulletin of the Michigan Fish Commission, 7: 1-73. In Biennial Report, Michigan State Board Fish Commission, I6(1903 1904).

REIGHARD, J. E., 1910. Methods of studying the habits of fishes, with an account of the breeding habits of the horned dace. Bulletin of the Cinited Stales Fish Commission, 28(2): 11111136.

REISMAN, H. M., 1963. Reproductive behavior of Apeltes quadracus, including some comparisons with other gasterosteid fishes. Copeia, 1963́: 191192.

REISMAN, H. M. \& CADE, T. J., 1967. Physiological and behavioral aspects of reproduction in the brook stickleback, Culaea inconstans. American Midland Naturalist, 77: 257 295.

RIBBINK, A. J., 1971. The behaviour of Hemihaplochromis philander, a South African cichlid fish. Zoologica Africana, 6: 263288

RIEDEL, K., 1914. Uber Gardaseefische, namentlich die Fortplanzung der Flussgrundel (Gobius fluvatilis Bonelli). Blätter für Aquarien- und Terrarienkunde, 25: 387 390. (As cited by Breder \& Rosen, 1966.)

ROBERTS, T. R., 1971. The fishes of the Malaysian family Phallostethidae (Atheriniformes). Brevinra, 374: 1-27.

ROBERTSON, D. R., 1973. Field observations on the reproductive behaviour of a pomacentrid fish, Acanthochromis polyacanthus. Zeitschrift für Tierpsychologie, 32: $319-324$.

ROWLAND, W. J., 1974. Reproductive behavior of the fourspine stickleback, Apeltes quadracus. Copeia, 1974: 183194.

RUCK, J. G., 1971. Development of the lumpfish, Trachelochismus melobesia (Pisces: Gobiesocidae). Zoological Publications from Victoria University of Wellington, 57: 19.

RUC.K, J. G., 1973a. Development of Triplerygion capito and T. robustum (Pisces: Tripterygiidae). Zoological Publications from Victoria Lniversity of Wellington, 63: 1 - 10. 
RUCK, J. G., 1973b. Development of the clingfishes, Diplocrepis puniceus and Trachelochismus pinnulatus (Pisces: Gobiesocidae). Zoological Publications from Victoria University of Wellington, 64: 1-12.

RUSSELL, B. C., 1971. Underwater observations on the reproductive activity of the demoiselle Chromis dispilus (Pisces: Pomacentridae). Marine Biology, Berlin, 10: 22-29.

RUWET, J.-C., 1962. Remarques sur le comportement de Tilapia macrochir Blgr. et T. melanopleura Dum. (Pisces: Gichlidae). Annales de la Société Royale Zoologique de Belgique, 92: 171-177.

RUWET, J.-C., 1963. Observations sur le comportement sexuel de Tilapia macrochir Blgr. (Pisces: Cichlidae) au lac de retenue de la Lufira (Katanga). Behaviour, 20: 242-250.

RYDER, J. A., 1886. The development of the toad-fish. American Naturalist, 20: 77-80.

SALE, P. F., 1970. Behaviour of the humbug fish. Australian Natural History, 16: 362-366.

SALE, P. F., 1971. The reproductive behaviour of the pomacentrid fish, Chromis caeruleus. Zeitschrift fiur Tierpsychologie, 29: 156-164.

SAVAGE, T., 1963. Reproductive behavior of the mottled sculpin, Cottus bairdi Girard. Copeia, 1963: 317-325.

SCHAPITZ, W., 1962. Das Verhalten von Pyrrhylina vittata (Regan) (Teleostei, Characidae). Zeitschrift für Tierpsychologie, 19:262 275.

SCOTT, D. B. C. \& FULLER, J. D., 1976. The reproductive biology of Scleropages formosus (Müller and Schlegel) (Osteoglossomorpha, Osteoglossidae) in Malaya, and the morphology of its pituitary gland. Journal of Fish Biology, 8: 45-53.

SENTA, T. \& WADA, I., 1970. The reproductive behavior in the gobiid fish Glossogobius olivaceus Temminck and Schlegel in an aquarium [in Japanese, English Summary]. Japanese Journal of Ichthyology, 17: 7-13.

SHAW, E., 1971. Evidence of sexual maturation in young adult shiner perch, Cymatogaster aggregata Gibbons (Perciformes, Embiotocidae). American Museum Novitates, 2479: 1-10.

SHIOGAKI, M. \& DOTSU, Y., 1973. The spawning behavior of the tripterygiid blenny, Tripterygion etheostoma [in Japanese, English Summary]. Japanese Journal of Ichthyology, 20: 36-41.

SMITH, C. L., ATZ, E. H. \& TYLER, J. C., 1971. Aspects of oral brooding in the cardinalfish Cheilodipterus affinis Poey (Apogonidae). American Museum. Novitates, 2456: 1-11.

SMITH, C. L., RAND, C. S., SCHAEFFER, B. \& ATZ, J. W., 1975. Latimeria, the living coelacanth, is ovoviviparous. Science, $\mathcal{N} . r_{\text {., }} 190: 1105-1106$.

SMITH, H. M., 1933. Contributions to the ichthyology of Siam. VII. The featherback fish Nolopterus chitala in Siam, with notes on its egg-laying and young. Journal of the Siam Society, Natural History Supplement 9: 245-258.

SMITH, H. M. \& HARRON, L. G., 1904. Breeding habits of the yellow cat-fish. Bulletin of the Uniled States Fish Commission, 22: 149-154.

SMITH, W. A., 1885. Notes on the sucker fishes, Liparis and Lepadogaster. Proceedings of the Royal Physical Society of Edinburgh, 9(1885-1888): 143-151.

STERBA, G., 1963. Freshwater Fishes of the World (Translated from German by D. W. Tucker). New York: Viking Press.

SWERDLOFF, S. N., 1970. Behavioral observations on Eniwetok damselfishes (Pomacentridae: Chromis) with special reference to the spawning of Chromis caeruleus. Copeia, 1970:371-374.

TAKAI, T. \& FUKUNAGA, T., 1971. The life history of an ovo-viviparous scorpaenoid fish, Sebastes longispinis (Matsubara)-I. Egg and larval stages [in Japanese, English Summary]. Journal of the Shimonoseki Universily of Fisheries, 20: $91-95$.

TAUTZ, A. F. \& GROOT, C., 1975. Spawning behavior of chum salmon (Oncorhynchus keta) and rainbow trout (Salmo gairdneri). Journal of the Fisheries Research Board of Canada, 32: 633-642.

TAYLOR, M., 1913. The development of Symbranchus marmoralus. Quarterly fournal of Microscopical Science, 59: $1-52$.

TAYLOR, W. R., 1969. A revision of the catfish genus Noturus Rafinesque, with an analysis of higher groups in the Ictaluridae. Bulletin of the United States National Museum, 282; 1-315.

TCHERNIAEV, G. A., 1971. La biologie du developpement de Comephorus dybowskii Kor. poisson endémique du Lac Baïkal. Bulletin de la Société Zoologique de France, 96: $385-398$.

THOMPSON, W. F., 1919. The spawning of the grunion (Leuresthes tenuis). Fish Bulletin, California Fish and Game Commission, 3: 129.

TIMMS, A. M. \& KEENLEYSIDE, M. H. A., 1975. The reproductive behaviour of Aequidens paraguayensis (Pisces: Cichlidae). Zeitschrift für Tierpsychologie, 39: 8-23.

TRIPLETT, E. L., 1960. Notes on the life history of the barred surf-perch, Amphistichus argenteus Agassiz, and a technique for culturing embiotocid embryos. California Fish and Game, 46: 433-439.

TURNER, C. H. \& EBERT, E. E., 1962. The nesting of Chromis punctipinnis (Cooper) and a description of their eggs and larvae. California Fish and Game, 48: 243-248.

VILLADOLID, D. V. \& MANACOP, P. R., 1934. The Philippine Phallostethidae, a description of a new species, and a report on the biology of Gulaphallus mirabilis Herre. Philippine Journal of Science, 55: 193-217.

VIPAN, J. A. M., 1886. On the nesting of a South-American siluroid fish (Callichthys littoralis) in this country [letter to Dr. Sclater]. Proceedings of the Zoological Society of London, 1886: 330-331.

WALLACE, C. R., 1972. Spawning behavior of Ictalurus natalis (LeSueur). Texas Journal of Science, 24: $307-310$.

WARD, J. A. \& BARLOW, G. W., 1967. The maturation and regulation of glancing off the parents by young orange chromides (Etroplus maculatus: Pisces-Cichlidae). Behaviour, 29: 1-56. 
WARD, J. A. \& WYMAN, R. L., 1975. The cichlids of the resplendent isle. Oceans, 8: 42-47.

WARD, J. A. \& WYMAN, R. L., 1977. Ethology and ecology of cichlid fishes of the genus Etroplus in Sri Lanka: preliminary findings. Enitronmental Biology of Fishes, 2: 137145.

WEBER, M., 1910. A new case of parental care among fishes. Proceedings, Section of Sciences, Koninklijke Nederlandse Akademie van Welenschappen, 13: 583-587.

WELCOMME, R. L., 1967. The relationship between fecundity and fertility in the mouthbrooding cichlid fish Tilapia leucosticta. Journal of Zoology, London, 151: $453-468$.

WERNER, Y. L., 1976. Notes on reproduction in the mouth-brooding fish Haplochromis flaviijosephi (Teleostei: Cichlidae) in the aquarium. Journal of Natural History, 10:669-680.

WESTIN, L.. 1969. The mode of fertilization, parental behaviour and time of egg development in fourhorn sculpin, Myoxocephalus quadricornis (L.). Report, Institute of Freshwater Research, Drottningholm, 49: 175182.

WESTIN, L., 1970. Observations on the nest digging of fourhorn sculpin Myoxocephalus quadricornis (L.). Report, Institute of Freshwater Research, Drotiningholm, 50:211-214.

WHITEHEAD, R. A., 1962. The life history and breeding habits of the West African cichlid fish Tilapia mariae and the status of T. meeki. Proceedings of the Zoological Society of London, 139: 535543.

WICKLER, W., 1962. "Egg dummies" as natural releasers in mouth-breeding cichlids. Nature, London, 194: $1092 \cdot 1093$.

WICKLER, W., 1965. Signal value of the genital tassel in the male Tilapia macrochir Blgr. (Pisces: Cichlidae). Nature, London, 208: 595596.

WICKLER, W., 1969. Zur Soziologie des Brabantbuntbarsches, Tropheus moorei (Pisces, Cichlidae). Zeitschrift für Tierpsychologie, 26: 967987.

WIF.BE, J. P., 1968. The reproductive cycle of the viviparous seaperch, Cymatogaster aggregata Gibbons. Canadian Journal of Zoology, 46: 1221 1234

WILLEY, A., 1909a. Observations on nests, eggs, and larvae of Ophiocephalus striatus. Spolia Zeylanica, 6: 108-123.

WILLEY, A., 1909b. The occurrence of Solenostoma of the coast of Ceylon. Spolia Zeylanica, 6: 102-107.

WOO'TON, R. J., 1971. A note on the nest-raiding behavior of male sticklebacks. Canadian Journal of Zoology, 49: $960-962$.

WOOTTON, R. J., 1972. The behaviour of the male three-spined stickleback in a natural situation: a quantitative description. Behaviour, 41:232-241.

WOOTTON, R. J., 1976. The Biology of the Sticklebacks. New York: Academic Press.

WOURMS, J. P. \& BAYNE, O., 1973. Development of the viviparous brotulid fish, Dinematichthys ilucoeteoides. Copeia, 1973: 3240.

WOURMS, J. P. \& COHEN, D. M., 1975. Trophotaeniae, embryonic adaptations, in the viviparous ophidioid fish, Oligopus longhursti: a study of museum specimens. Journal of Morphology, 147: 385402.

WOURMS, J. P. \& EVANS, D., 1974. The annual reproductive cycle of the black prickleback, Xiphister atropurpureus, a Pacific coast blennioid fish. Canadian fournal of Zoology, 52: 795802.

WYMAN, J., 1859. On some unusual modes of gestation. American Journal of Science and Arts, 27: 5 13.

YAPCHIONGCO, J. V., 1963. Spawning habits of the murrell or snakehead fish, Ophicephalus striatus Bloch. Copeia, 1963: 700702

\section{INDEX TO NAMES IN TABLES 2-10}

Acipenseriformes, 10

Adrianichthyidac, 9,11

Alabetidae, 7,12

Amblyopsidae, 9, 11

Amiidae, 5, 10

Amiiformes, 10

Amphipnoidae, 6,12

Anabantidae, 6,12

Anablepidae, 9, 11

Anarhichadidae, 7, 12

Anguilliformes, 10

Antennariidae, 5, 11

Aphredoderidae, 7,11

Apogonidae, 7, 12

Ariidae, 7,11

Aspredinidae, 9, 11

Atheriniformes, 11

Aulorhynchidac, 5, 12

Bagridae, 7, 11

Balistidac, 7, 12
Batrachoididae, 5, 11

Batrachoidiformes, 11

Belontiidae, 7,12

Beryciformes, 11

Blenniidae, 6,12

Callichthyidae, 5, 11

Carangidac, 10, 12

Centrarchidae, 6,12

Cetomimiformes, 11

Channidae, 7,12

Channiformes, 12

Characidae, 8,11

Cichlidae, 8,12

Githarinidae, 5, 11

Clariidac, 5, 11

Clinidae, 7,12

Clupeiformes, I1

Clupeomorpha, 11

Coelacanthiformes, 10

Comephoridae, 9, 12 
Cottidae, 6, 12

Ctenothrissiformes, 11

Cyclopteridae, 6, 12

Cyprinidae, 5, 11

Cypriniformes, 11

Cyprinodontidae, 5, 11

Dactylopteriformes, 12

Dipteriformes, 10

Elopiformes, 10

Elopomorpha, 10

Embiotocidae, 9, 12

Erythrinidae, 5, 11

Euteleostei, 11

Exocoetidae, 9, 11

Gradiformes, 11

Gadopsidae, 10, 12

Galaxiidae, 7,11

Gasterosteidae, 5, 12

Gasterosteiformes, 12

Gobiesocidae, 6, I1

Gobiesociformes, 11

Gobiidae, 8, 12

Gonorynchiformes, 11

Goodeidae, 9, 11

Gymnarchidae, 10

Gymnotidae, 5, 11

Hemiodontidae, 10, 11

Heteropneustidae, 7, 11

Hexagrammidae, 5, 12

Ictaluridae, 8, 11

Jenynsiidae, 9, 11

Kurtidae, 6, 12

Labridae, 6, 12

Lampridiformes, 12

Latimeriidae, 9, 10

Lebiasinidae, 5,11

Lepidosirenidae, 5,10

Lophiiformes, 11

Loricariidae, 5,11

Luciocephalidae, 10, 12

Malapteruridae, 10, 11

Mormyriformes, 10

Nandidae, 6, 12
Notacanthiformes, 10

Notopteridae, 5,10

Nototheniidae, 7, 12

Ophidiidae, 9, 11

Opisthognathidae, 6, 12

Oryziatidae, 9, 11

Osmeridae, 9, 11

Osphronemidae, 7, 12

Osteoglossidae, 8, 10

Osteoglossiformes, 10

Osteoglossomorpha, 10

Pantodontidae, 5, 10

Pegasiformes, 12

Percidac, 7, 12

Perciformes, 12

Percopsiformes, 11

Pholididae, 9, 12

Plesiopidae, 6, 12

Plcuronectiformes, 12

Plotosidae, 5, 11

Poeciliidae, 9, 11

Polypteridae, 10

Polypteriformes, 10

Pomacentridae, 6, 12

Pseudochromidae, 6, 12

Salmonidae, 9, 11

Salmoniformes, 11

Scorpaenidae, 9, 12

Scorpaeniformes, 12

Semionotiformes, 10

Serranidae, 7, 12

Siluridae, 5, 11

Siluriformes, 11

Solenostomidae, 9,12

Sparidae, 6, 12

Stichacidae, 7, 12

Synbranchidae, 5, 12

Synbranchiformes, 12

Syngnathidae, 5, 12

Tetraodontidae, 6, 12

Tetraodontiformes, 12

Theraponidae, 6, 12

Tripterygiidae, 6,12

Umbridae, 7, 11

Zeiformes, 11

Zoarcidae, 9, 11 